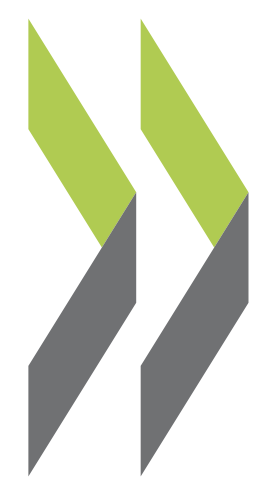

OECD Science, Technology and Industry Working Papers $2015 / 05$

The Use of Patent Statistics for International Comparisons and Analysis of Narrow Technological

Ivan Haščič, Jérôme Silva, Nick Johnstone

Fields 


\section{OECD SCIENCE, TECHNOLOGY AND INDUSTRY WORKING PAPERS}

OECD Working Papers should not be reported as representing the official views of the OECD or of its member countries. The opinions expressed and arguments employed are those of the authors.

Working Papers describe preliminary results or research in progress by the author(s) and are published to stimulate discussion on a broad range of issues on which the OECD works. Comments on Working Papers are welcomed, and may be sent to OECD Directorate for Science, Technology and Innovation, OECD, 2 rue André-Pascal, 75775 Paris Cedex 16, France; e-mail: sti.contact@oecd.org.

The release of this working paper has been authorised by Andrew Wyckoff, OECD Director for Science, Technology and Innovation.

This paper has been authored by Ivan Haščič, Jérôme Silva (OECD Environment Directorate) and Nick Johnstone (OECD Directorate for Science, Technology and Innovation). The paper draws on past work of the Environment Directorate and its project on "Environmental policy and technological innovation" (http://www.oecd.org/environment/innovation.htm).

A version of this paper has been reviewed by the OECD Working Party on Environmental Information (WPEI) at its meeting in November 2014 and has benefited from the comments received. The authors are grateful to Dominique Guellec and Hélène Dernis for helpful comments on a previous version of this paper, as well as the participants of the Conference on Patent Statistics for Decision Makers (October 2013 in Rio de Janeiro, Brazil) and the Workshop on Evaluating Green Innovation Policies (December 2012 at Bruegel Institute in Brussels, Belgium) where previous versions of this paper were presented.

The statistical data for Israel are supplied by and under the responsibility of the relevant Israeli authorities. The use of such data by the OECD is without prejudice to the status of the Golan Heights, East Jerusalem and Israeli settlements in the West Bank under the terms of international law.

a) Note by Turkey:

The information in this document with reference to "Cyprus" relates to the southern part of the Island. There is no single authority representing both Turkish and Greek Cypriot people on the Island. Turkey recognises the Turkish Republic of Northern Cyprus (TRNC). Until a lasting and equitable solution is found within the context of the United Nations, Turkey shall preserve its position concerning the "Cyprus issue".

b) Note by all the European Union Member States of the OECD and the European Union:

The Republic of Cyprus is recognised by all members of the United Nations with the exception of Turkey. The information in this document relates to the area under the effective control of the Government of the Republic of Cyprus.

\section{(c) OECD/OCDE 2015}

Applications for permission to reproduce or translate all or part of this material should be made to: OECD Publications, 2 rue André-Pascal, 75775 Paris, Cedex 16, France; e-mail: rights@oecd.org 


\title{
THE USE OF PATENT STATISTICS FOR INTERNATIONAL COMPARISONS AND ANALYSIS OF NARROW TECHNOLOGICAL FIELDS
}

\author{
by Ivan Haščič, Jérôme Silva and Nick Johnstone (OECD)
}

\begin{abstract}
Patent data provide an increasingly used means to analyse innovation performance worldwide including in countries with incomplete data coverage, such as some developing countries. This paper discusses the specific issues associated with using patent data for measuring and analysing innovation in narrow technological fields, such as many environment-related technologies. To improve crosscountry comparability of patent statistics, the paper advocates the use of indicators based on patent family size because they are more flexible and can be adapted to various applications. The paper also examines certain idiosyncratic characteristics of patent databases and proposes approaches to mitigate potential biases in empirical cross-country analyses. While doing so is particularly important for analyses of narrow technological fields such as many environment- and climate-related technologies, some of these issues are relevant for patent analysis more broadly.
\end{abstract}

Keywords: innovation, indicators, environmental technologies

JEL classification: O3; O31; O34; Q2; Q4; Q5 


\section{EXECUTIVE SUMMARY}

Patent data provide an increasingly used means to analyse innovation performance worldwide including in countries with incomplete data coverage, such as some developing countries. This paper focuses on the construction of indicators and analytical methodologies for measuring innovation in climate- and other environment-related technologies. A companion paper discusses the use of such indicators for policy purposes (see Haščič and Migotto 2015).

Various approaches have previously been developed to improve cross-country comparability of patent statistics. However, they were generally limited either because they require additional data that might not be available in all countries or because they impose restrictions that are less appropriate for analysis of narrow technological fields. The simultaneity of two conditions - narrow technological scope and comparability across a wide range of countries - often renders existing indicators inapt for such analyses. This paper advocates the use of indicators based on patent family size because they are more flexible and can be adapted to such applications.

This paper also examines certain idiosyncratic characteristics of patent databases. The sources of such idiosyncrasies include unequal country and temporal coverage of patent databases, asymmetric information on patent protection in member states through regional applications, missing descriptive information in patent databases, and non-homogeneous assignment of patent classes and the consequent difficulty of identifying relevant patent documents. This suggests that all patent databases should be exploited with care. The paper proposes approaches to mitigate potential biases in empirical cross-country analyses. While doing so is particularly important for analyses of narrow technological fields such as environment and climate related technologies, many of these issues are relevant for patent analysis more broadly. 
TABLE OF CONTENTS

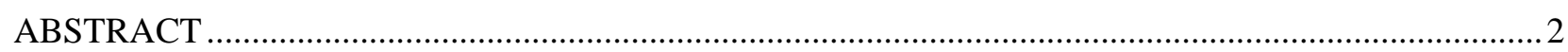

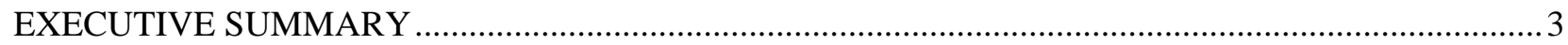

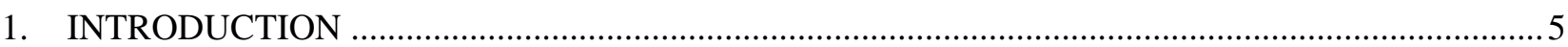

2. ADAPTING PATENT INDICATORS TO DIFFERENT CONTEXTS …...........................................

3. IDIOSYNCRATIC ISSUES IN CONSTRUCTION AND ANALYSIS OF PATENT

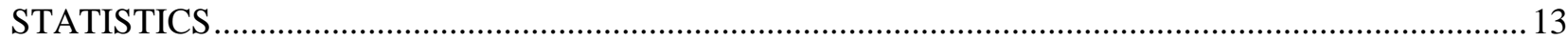

3.1 Country and temporal coverage of patent databases..............................................................13

3.2 Protection in national jurisdictions through regional (international) patent filings ........................24

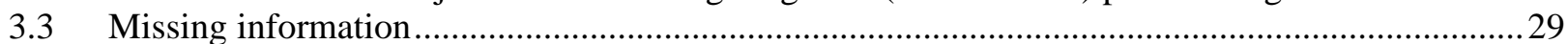

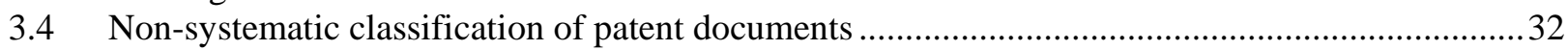

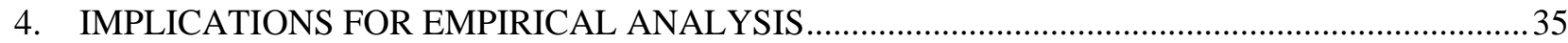

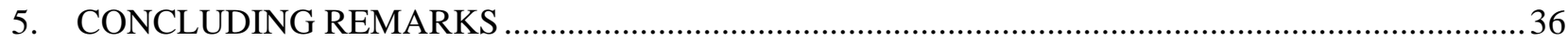

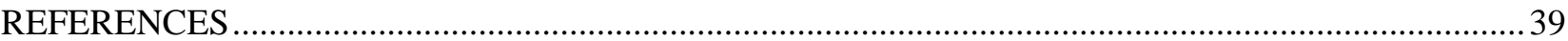

ANNEX 


\section{INTRODUCTION}

Patent data provide an increasingly used means to analyse innovation performance worldwide including in countries with incomplete data coverage, such as some developing countries. This paper focuses on the construction of indicators and analytical methodologies for measuring innovation in climate- and other environment-related technologies. A companion paper discusses the use of such indicators for policy purposes (see Haščič and Migotto 2015).

More generally, there is a growing demand for analysis of patenting and innovation performance worldwide including in countries with incomplete data coverage, such as some developing countries (see e.g., Collier and Venables 2012; Belward et al. 2011; Barton 2007). However, past analyses have not properly taken into account the varying geographic coverage of patent databases (including those by the authors of this paper). This paper builds on the first attempts to reflect upon this issue in Haščič et al. (2012). Although the sources of idiosyncrasy are discussed in the context of the EPO's PATSTAT database, similar approaches could be used to mitigate biases arising out of other patent databases.

In addition, patent data are increasingly used for international comparisons and analysis in relatively 'narrow' fields of technology, such as many environment-related technologies (see e.g. OECD 2011, 2012; Dechezleprêtre et al. 2011). However, achieving both comparability across countries and representativity within a country (statistical robustness) creates additional challenges. Although several approaches have been developed to improve cross-country comparability of patent statistics, these are not always suitable for such analyses - either because they require additional data that might not be available in all countries or because they impose restrictions that are less appropriate for analysis of narrow technological fields. The simultaneity of these two conditions - narrow technological scope and comparability across a wide range of countries - often renders many existing indicators inapt for such analyses.

This paper first discusses why different patent indicators might be more or less suitable to achieve the two objectives simultaneously. It then advocates the use of indicators based on patent family size that are more flexible and can be adapted for such applications, provided that certain idiosyncratic characteristics of patent databases are addressed. The following section discusses in detail these idiosyncratic characteristics of patent databases that are of general relevance (unequal country and temporal coverage of patent databases, asymmetric information on designation of member states in regional and PCT applications) as well as those that are of relevance particularly for narrow technological fields (missing information, identification of relevant patent documents). The paper proposes approaches to mitigating potential biases arising out of such idiosyncrasies - both in construction of patent statistics (treatment of underlying data) as well as in empirical analyses that use such statistics. In particular, it advocates the use of a robust control variable (e.g. TOTPAT) for crosscountry analyses of narrow technological fields. All methodological issues discussed here and examples provided are based on the PATSTAT database, but could be applied to patent databases more generally. 


\section{ADAPTING PATENT INDICATORS TO DIFFERENT CONTEXTS}

Many of the existing patent indicators, including counts of patent applications registered at a given national or regional patent office, Patent Cooperation Treaty (PCT) applications, or the triadic patent family (TPF) indicator, have one or both of the following shortcomings: i) either they are not suitable for international comparisons; and/or b) they are less suitable for analysis of narrow technological fields. The joint existence of two requirements on the indicators - comparability across countries and statistical robustness - often renders these indicators inappropriate for many applications. The reasons range from a home bias in national and regional filings, differences in propensities to use the PCT route (a special type of a home bias) ${ }^{1}$, to an overly restrictive quality threshold applied precisely to address cross-country differences in patent quality (such as the TPF). Moreover, much of the complementary data that is commonly used to assess patent value (e.g. citation data) are currently available only in a very limited number of countries.

To illustrate the differences, Figures 1-3 give examples of alternative patent indicators for technological fields of different 'thickness' and for different geographical scales. Four alternative indicators are shown - PCT applications, TPF applications, "claimed priorities" (equivalent to patent family size $>=2$, or PF2) and all priorities (equivalent to patent family size $>=1$, or PF1). ${ }^{2}$

Given our reliance upon data obtained from a source which does not include the full population of patented inventions within a given field (see below) we do not have any external measure against which we can assess which statistic is optimal. However, in order to assess the reliability of the use of alternative statistics as indicators of inventive activity, and their suitability for empirical analysis it is interesting to compare: a) the degree of correlation between alternative measures; b) the extent of variation within fields over time and across countries; and, c) the presence of zero values.

To measure inventive activity worldwide in relatively 'thick' fields - several of the indicators seem satisfactory (for instance, see renewable energy and water pollution abatement in Figure 1). The different measures are highly correlated, and at the country-level the variation is "plausible" for most countries. The situation changes with a focus on narrower technological fields (e.g. wind power, geothermal energy) which compromises performance of the most restrictive versions of these indicators. For example, in the case of geothermal energy, the frequency of zero counts is so high that it limits the information value of the TPF indicator (Figure 1).

These problems are accentuated when the geographic scope is reduced. Focusing on individual countries results in high frequency of zero counts for all but the largest inventor countries (Figures 23) Indeed, even for thick fields some of the more restrictive counts (e.g. TPF and PF2) give relatively low variation at the country level (Figures 2 and 3).

In general, PF2 seems as a suitable compromise between comparability (quality threshold) and practicality (variance), except for very narrow technological fields or small inventor countries - in which case PF1 might be the only option.

In sum, while restricting patent family size is a useful way to impose a certain quality threshold across countries (Dernis et al. 2001), the TPF condition of a specific EP-US-JP triad (Dernis and Khan 2004) is overly restrictive and potentially biased for the contexts discussed here. In such cases, it might be better to relax this restriction and instead adjust it depending on the application. While for some applications no restriction will be the only option (PF1), for others the dyadic (any office, or PF2) restriction might be feasible, and cases of particularly "thick" technological fields or broad geographic scopes will permit imposing the triadic (any office, or PF3) restriction, or higher. For these 
reasons, this paper advocates the use of a range of patent family indicators, as a generalization of the TPF concept.

It must be noted that PF3 differs from the TPF indicator in two respects - (i) the TPF requires a specific triad (EP-US-JP) while PF3 can include any triad; (ii) the TPF is based on an extended patent family while PF3 is based on simple patent family. It would be a natural extension of the TPF concept to define a "dyadic patent family" (DPF) indicator as a dyad, perhaps any dyad of two offices. This would however require addressing the problem of regional and international patent filings (see Section 3 for a discussion). 
The Use of Patent Statistics for International Comparisons and Analysis of Narrow Technological Fields

Figure 1. Patent indicators in different contexts: Technological scope
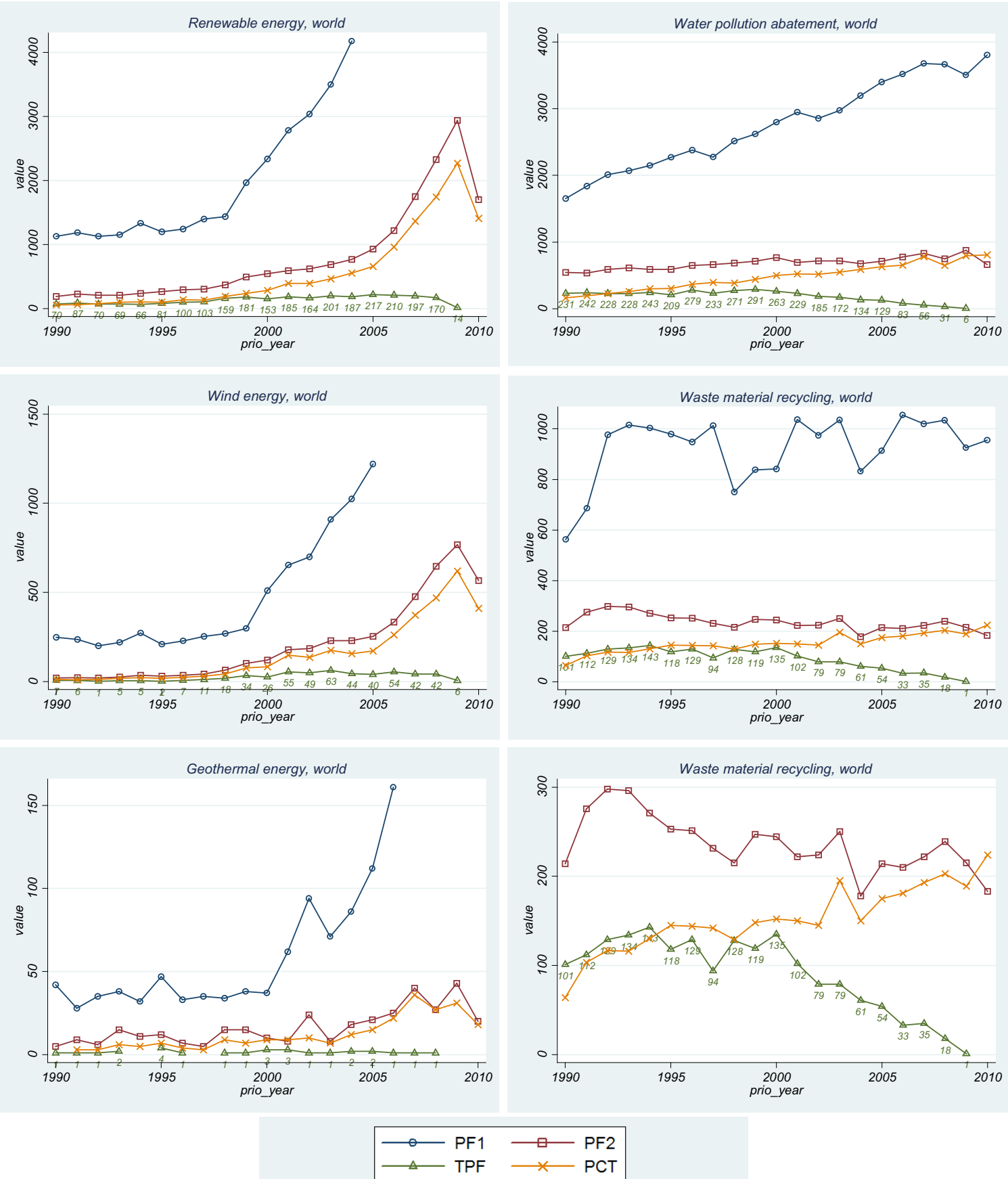

Note: Figures in the left-hand panel are dominated by PF1; therefore the $y$-axis is reduced cutting off PF1 values for later years in the time series to allow better visibility of the remaining series. 
The Use of Patent Statistics for International Comparisons and Analysis of Narrow Technological Fields

Figure 2. Patent indicators in different contexts: Inventor country
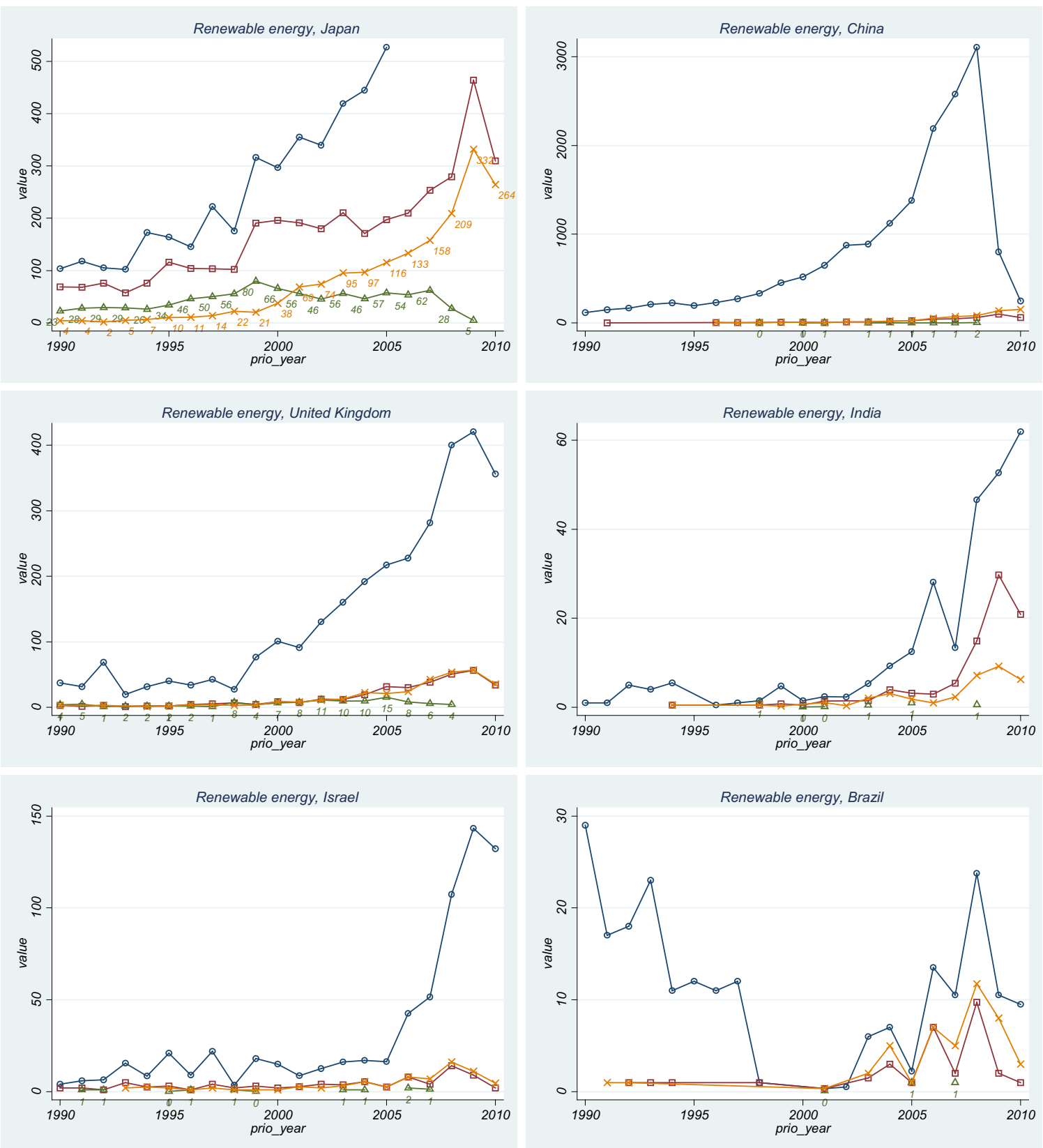

$$
\begin{array}{lll}
\longrightarrow & \text { PF1 } & \square \\
\square & \text { PF2 } \\
\square & \text { TPF } & \longrightarrow
\end{array}
$$


The Use of Patent Statistics for International Comparisons and Analysis of Narrow Technological Fields

Figure 3. Patent indicators in different contexts: Inventor country
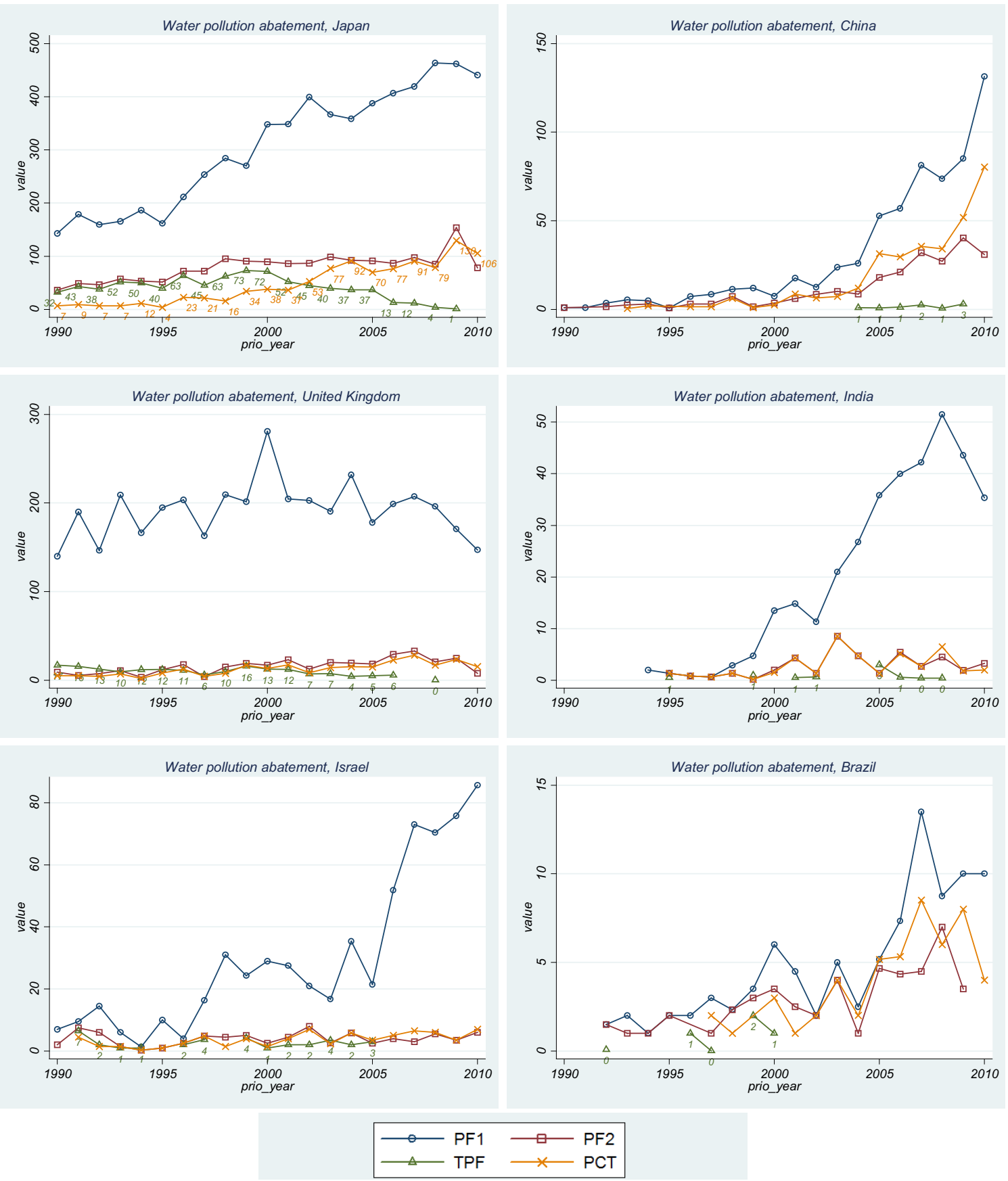
Table 1 summarizes the proposed range of indicators and places them in the context of the existing indicators.

Table 1. Patent indicators

\begin{tabular}{|c|c|c|c|}
\hline Indicator & Definition & Main strengths and weakness & Purpose \\
\hline National applications & $\begin{array}{l}\text { Singletons, claimed priorities } \\
\text { and duplicates }\end{array}$ & $\begin{array}{l}\text { Commensurate in terms of patent } \\
\text { breadth and quality; subject to a } \\
\text { home bias. }\end{array}$ & \multirow{3}{*}{$\begin{array}{l}\text { Measures of } \\
\text { patenting } \\
\text { activity } \\
\text { (based on } \\
\text { counting } \\
\text { applications } \\
\text { or grants) }\end{array}$} \\
\hline Regional applications & $\begin{array}{l}\text { Singletons, claimed priorities } \\
\text { and duplicates }\end{array}$ & $\begin{array}{l}\text { As above; difficulty with } \\
\text { designation of member states. }\end{array}$ & \\
\hline PCT applications & $\begin{array}{l}\text { Singletons, claimed priorities } \\
\text { and duplicates }\end{array}$ & $\begin{array}{l}\text { Subject to its own kind of 'home } \\
\text { bias'; propensity to use the PCT } \\
\text { route varies across countries. }\end{array}$ & \\
\hline $\begin{array}{l}\text { Triadic patent family } \\
\text { (TPF) }\end{array}$ & $\begin{array}{l}\text { Triad (incl. EP, JP, US } \\
\text { grants); based on extended } \\
\text { patent family. }\end{array}$ & $\begin{array}{l}\text { Only high-quality inventions; too } \\
\text { restrictive for narrow tech fields. }\end{array}$ & \multirow{4}{*}{$\begin{array}{l}\text { Measures of } \\
\text { inventive } \\
\text { activity } \\
\text { (based on } \\
\text { counting } \\
\text { patent } \\
\text { families) }\end{array}$} \\
\hline PF1 - All priorities & $\begin{array}{l}\text { Family size }>=1 \text {, any office, } \\
\text { based on simple patent } \\
\text { family (singletons and } \\
\text { claimed priorities). }\end{array}$ & $\begin{array}{l}\text { A complete picture of inventive } \\
\text { activity worldwide because the } \\
\text { entire stock of patent priorities is } \\
\text { considered; too lax because it } \\
\text { gives equal weight to low- and } \\
\text { high-quality inventions; does not } \\
\text { account for differences in patent } \\
\text { breadth across offices. }\end{array}$ & \\
\hline PF2 - Claimed priorities & $\begin{array}{l}\text { Family size }>=2, \text { any office, } \\
\text { based on simple patent } \\
\text { family. }\end{array}$ & $\begin{array}{l}\text { A limited quality threshold suitable } \\
\text { for many narrow tech fields. }\end{array}$ & \\
\hline PF3 & $\begin{array}{l}\text { Family size }>=3 \text {, any office, } \\
\text { based on simple patent } \\
\text { family. }\end{array}$ & $\begin{array}{l}\text { Only high-quality inventions, but } \\
\text { less restrictive than the TPF. }\end{array}$ & \\
\hline
\end{tabular}

For the purpose of international comparisons, patent statistics based on counting distinct patent families (patent priorities) are preferable for several reasons: (i) considering only priority applications (and not their duplicates) avoids double-counting - which would occur if data from multiple patent offices were pooled. The data is thus better suited for cross-country analysis; (ii) the data are truly world-wide in their coverage - and thus less subject to bias - because the entire stock of patent priorities is considered (see also de Rassenfosse et al. 2013). ${ }^{4}$

In addition, a subset of these statistics - those based on counting only the 'claimed priorities' (i.e. patent priorities that have been 'claimed' as a priority by another patenting filing abroad) provides a quality threshold because priority applications which have never been claimed (singletons) are excluded. This helps contain concerns over low-value and strategic patenting. Counting the claimed priorities gives rise to the PF2 statistic (i.e. patent families with size 2 or greater).

It turns out that for many applications related to environmental technologies, the PF2 restriction (claimed priorities) is particularly suitable in order to achieve the dual purpose of comparability and robustness. The count of claimed priorities (i.e. patent applications deposited at any office world-wide, that have been claimed as priority elsewhere in the world) is a suitable statistic for the purpose of international comparisons because only the priority applications protecting "high-value" invention are 
counted. The reason that claimed priorities can be viewed as representing inventions of higher value is that patenting is costly (e.g. translation and maintenance fees). As such, a firm will only go abroad to protect its intellectual property if it expects that the commercial value of its invention justifies it. Previous research has shown that the number of additional patent applications (other than the priority application) is a good indicator of patent value (Guellec and van Pottelsberghe 2000; Harhoff et al. 2003). The results in Guellec \& van Pottelsberghe (2000) suggest that patent value increases up to family size of 4 (or 5), and decreases thereafter. However, few patent applications have family size greater than five.

The use of claimed priorities based on an economic threshold criterion was advocated already by Faust and Schedl 1983 and Faust 1990. For example, Faust (1990) argued that by excluding priority applications which have never been claimed abroad (singletons) this approach will exclude the large number of exclusively domestic Japanese patent applications with usually only one claim. ${ }^{5}$

Moreover, counts of claimed priorities (PF2 statistics) have the advantage that they do not suffer from biases due to changing publication practices at the USPTO. Prior to 2001, only grants (not applications) were published by the USPTO. Even after 2001, the rules allow applicants to opt-out from having their patent applications made public before grant (i.e. the non-publication request). These distinctions between grants and applications create biases in cross country comparisons when using the PF1 statistic. This is a limitation of using PF1 statistics compared with PF2, PF3, etc. that do not suffer from such bias.

The downside of counting claimed priorities is the duplication lag that weighs more on indicators with higher minimum family size. Another potential problem for determining family size is the question of how to treat applications filed through the regional (EP, AP) and international (PCT) routes. For example, should a regional application have an equal weight as a national application (e.g. treating EPO as a single jurisdiction), or should it rather be taken to represent individual member states? In the latter case how should the individual weights be determined? ${ }^{6}$ This is similar to the problem of 'nowcasting' PCT applications in international phase towards national/regional phase. And again, should PCT applications in national/regional phase have an equal weight as a national application, or should a weight be estimated? We propose a solution in Section 3.2 below.

While the PF2 is indeed often a good compromise between the too restrictive TPF and the too lax count of all priorities (PF1), in some circumstances PF1 can be the only practical approach - e.g. for very narrow technological fields or for analysis requiring a high degree of spatial disaggregation. This approach can be generalized by allowing the analyst to choose the degree of restriction - the number of unique countries (patent offices) within a simple patent family - the minimum 'quality' threshold that still allows for sufficient variation in the measured statistic (avoids too many zeros). The choice of the restriction is then a function of 'scope' (narrow vs thick) of the technological field analysed, geographic coverage, as well as country context (propensity to protect inventions abroad). However, construction of a reliable indicator also requires addressing a certain number of potential pitfalls associated with patent databases. They are discussed next. 


\section{IDIOSYNCRATIC ISSUES IN CONSTRUCTION AND ANALYSIS OF PATENT STATISTICS}

\subsection{Country and temporal coverage of patent databases}

Analyses using patent data have typically focused on OECD countries for which data availability is generally satisfactory. However, the recent growing interest in developing countries raises the importance of idiosyncrasies arising out of unequal database coverage which often varies highly among countries worldwide. Accounting for such idiosyncrasies is all the more important for analyses that study narrow technological fields because the high level of disaggregation leads to high frequency of zero (or very low) counts.

Ideally, one would need information on the representativeness of PATSTAT with respect to national registers. In the absence of such information, we use the EPO's weekly updates of the "contents and coverage of the DOCDB bibliographic file" - the DOCDB is the source database from which a 'snapshot' is drawn biannually and released as PATSTAT. The April 2012 release of PATSTAT includes batches of data provided from as many as 94 different patent offices, including all major patent offices worldwide. This is shown as cumulative coverage in Figure 4. However, for some offices the time series are incomplete and thus the contemporaneous coverage (for a given year) is typically somewhat lower. The apparent drop in coverage in recent years is most likely only a temporary phenomenon because some batches of new data are included with a lag. On-going efforts of the EPO to acquire historical batches of data would shift the curves upward, and bring them closer together.

Figure 4. Contemporaneous and cumulative data coverage in PATSTAT APR12

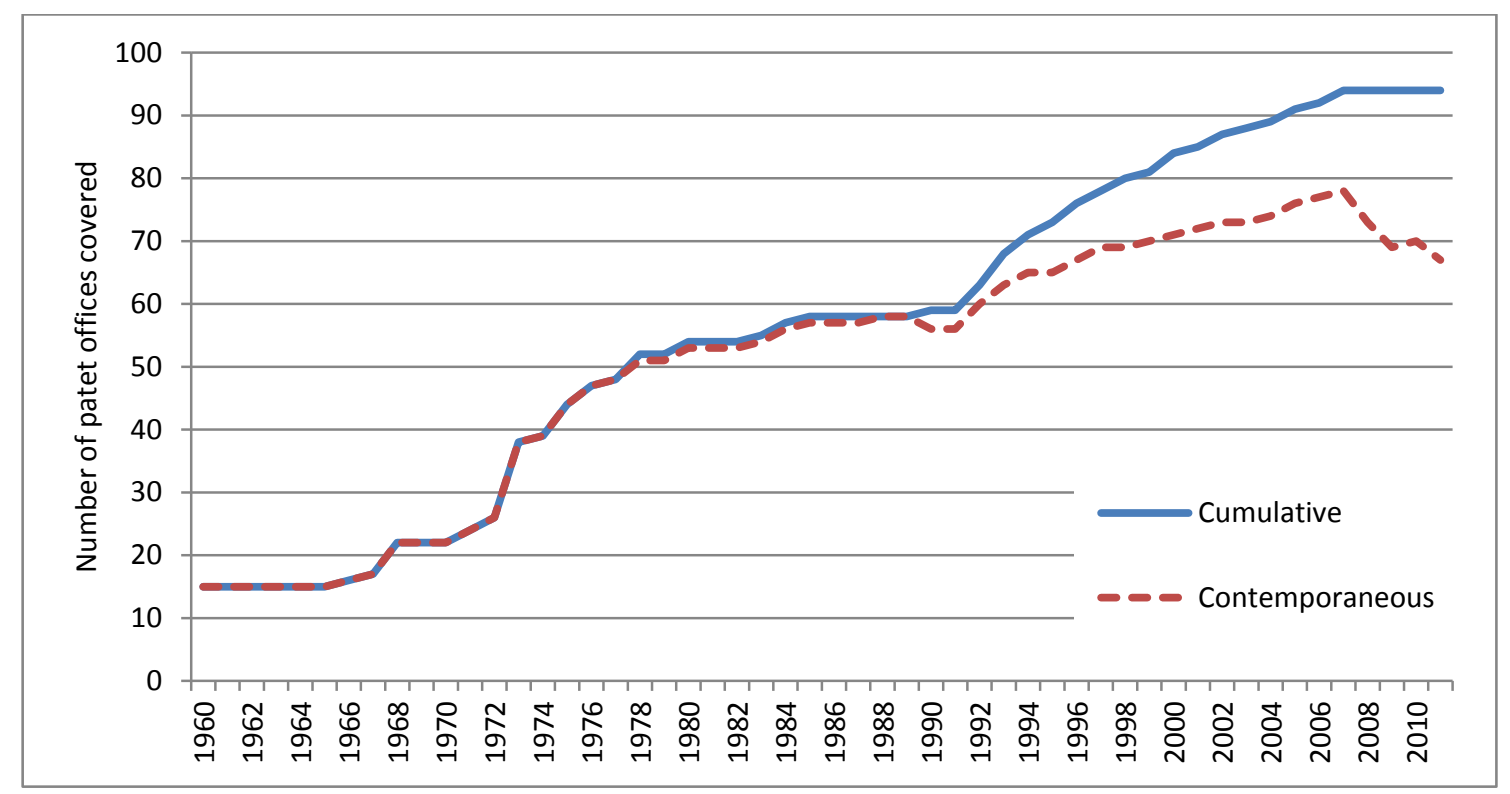

Note: Excluding data batches for utility models or petty patents.

The status of coverage in PATSTAT can be classified in four categories: (i) For 1980-2009 there are 39 offices with complete coverage during the entire 30-year period (e.g. JP, US, EP, IB); (ii) Further 16 offices have a complete coverage although defined for less than 30 years (e.g. DD, CS, $\mathrm{YU})^{7}$; (iii) Other 40 offices have only partial coverage of patent applications deposited in any given year, for instance only certain months in a year or only certain types of patent applications (e.g. AR, 
IN, MA); (iv) Finally, the remaining offices or countries have no coverage, theoretically, although for various reasons PATSTAT includes data for additional 19 offices (e.g. inclusion of data on prior art), meaning that there is data on an additional 129 inventor countries (Table 2).

Coverage of OECD countries is generally complete, although some gaps exist, notably for Chile (1980-2004 and 2009 are not covered at all, 2005 and 2008 only partly). Among non-OECD countries it is noteworthy that countries such as Brazil, South Africa, Egypt and Russia have complete coverage. However, gaps in coverage exist for some other countries, such as Argentina (1995-96 are only partly covered), India (2007 is only partly covered, 2008-09 not at all), China (1980-84 are not covered at all, 1985 only partly), Indonesia (1980-95 and 2002-09 are not covered at all, 1996-97 only partly) and Philippines (1999 only partly covered, 2000-09 not at all). 
Table 2. Theoretical and empirical data coverage in PATSTAT APR12

\begin{tabular}{|c|c|c|c|c|c|}
\hline \multirow{2}{*}{\multicolumn{2}{|c|}{ Country/office }} & \multirow{2}{*}{\multicolumn{2}{|c|}{$\begin{array}{c}\text { Theoretical coverage, } \\
\text { data batches for 1980-2009 }\end{array}$}} & \multicolumn{2}{|c|}{ Empirical coverage, 1980-2009 } \\
\hline & & & & \multirow{2}{*}{$\begin{array}{r}\text { as Application Authority(1) } \\
10,658,985\end{array}$} & \multirow{2}{*}{$\begin{array}{r}\text { as Inventor Country(2) } \\
3,908,024\end{array}$} \\
\hline $\mathrm{JP}$ & Japan & 30 & complete & & \\
\hline US & United States & 30 & complete & $5,449,125$ & $5,933,195$ \\
\hline EP & European Patent Office [EPO] & 30 & complete & $2,707,381$ & \\
\hline $\mathrm{DE}$ & Germany & 30 & complete & $2,574,630$ & $3,090,843$ \\
\hline KR & Korea (South) & 30 & complete & $1,921,667$ & $1,633,271$ \\
\hline$A U$ & Australia & 30 & complete & 982,172 & 144,914 \\
\hline $\mathrm{CA}$ & Canada & 30 & complete & 963,367 & 366,256 \\
\hline SU & Soviet Union (former) & 30 & complete & 766,954 & 544,381 \\
\hline AT & Austria & 30 & complete & 663,518 & 183,190 \\
\hline GB & United Kingdom & 30 & complete & 517,375 & 905,490 \\
\hline ES & Spain & 30 & complete & 516,678 & 155,283 \\
\hline FR & France & 30 & complete & 484,821 & $1,113,411$ \\
\hline BR & Brazil & 30 & complete & 408,144 & 74,490 \\
\hline DK & Denmark & 30 & complete & 192,652 & 126,214 \\
\hline$M X$ & Mexico & 30 & complete & 176,070 & 17,390 \\
\hline IT & Italy & 30 & complete & 165,372 & 509,374 \\
\hline PL & Poland & 30 & complete & 157,305 & 69,593 \\
\hline NO & Norway & 30 & complete & 150,368 & 70,045 \\
\hline IL & |srael 8 & 30 & complete & 132,758 & 127,959 \\
\hline$Z A$ & South Africa & 30 & complete & 132,370 & 23,184 \\
\hline SE & Sweden & 30 & complete & 116,434 & 332,107 \\
\hline $\mathrm{FI}$ & Finland & 30 & complete & 109,235 & 189,493 \\
\hline $\mathrm{NZ}$ & New Zealand & 30 & complete & 99,370 & 24,980 \\
\hline $\mathrm{HU}$ & Hungary & 30 & complete & 93,013 & 49,931 \\
\hline $\mathrm{NL}$ & Netherlands & 30 & complete & 89,231 & 383,733 \\
\hline PT & Portugal & 30 & complete & 87,305 & 8,962 \\
\hline IB & International Bureau of the WIPO & 30 & complete & 77,984 & - \\
\hline HK & Hong Kong, China & 30 & complete & 69,446 & 23,408 \\
\hline $\mathrm{CH}$ & Switzerland & 30 & complete & 66,335 & 380,553 \\
\hline $\mathrm{IE}$ & Ireland & 30 & complete & 46,544 & 34,043 \\
\hline TR & Turkey & 30 & complete & 43,447 & 27,571 \\
\hline RO & Romania & 30 & complete & 42,023 & 31,733 \\
\hline $\mathrm{BE}$ & Belgium & 30 & complete & 41,252 & 158,641 \\
\hline BG & Bulgaria & 30 & complete & 32,092 & 23,625 \\
\hline EG & Egypt & 30 & complete & 8,474 & 2,285 \\
\hline LU & Luxembourg & 30 & complete & 7,300 & 12,507 \\
\hline $\mathrm{CY}$ & Cyprus $^{9}$ & 30 & complete & 1,370 & 1,396 \\
\hline MC & Monaco & 30 & complete & 957 & 2,722 \\
\hline $\mathrm{LI}$ & Liechtenstein & & complete & - & 5,737 \\
\hline $\mathrm{AP}$ & African Regional IPO [ARIPO] ${ }^{3}$ & 25.2 & complete & 5,524 & - \\
\hline YU & Yugoslavia - Serbia/Montenegro & 23.3 & complete & 14,576 & 8,939 \\
\hline DD & Eastern Germany (former) & 19.5 & complete & 105,734 & 85,185 \\
\hline LT & Lithuania & 17.2 & complete & 3,745 & 2,484 \\
\hline SI & Slovenia & 17.1 & complete & 29,838 & 10,079 \\
\hline $\mathrm{RU}$ & Russian Federation & 16.9 & complete & 479,655 & 243,024 \\
\hline $\mathrm{CZ}$ & Czech Republic & 16.8 & complete & 67,030 & 42,134 \\
\hline SK & Slovak Republic & 16.2 & complete & 23,904 & 7,731 \\
\hline LV & Latvia & 15.8 & complete & 4,259 & 2,854 \\
\hline MD & Republic of Moldova & 15.6 & complete & 4,486 & 4,430 \\
\hline $\mathrm{HR}$ & Croatia & 15.4 & complete & 11,924 & 6,393 \\
\hline EE & Estonia & 14.0 & complete & 6,164 & 4,089 \\
\hline CS & Czechoslovakia (former) & 14.0 & complete & 78,257 & 49,765 \\
\hline
\end{tabular}


The Use of Patent Statistics for International Comparisons and Analysis of Narrow Technological Fields

\begin{tabular}{|c|c|c|c|c|c|}
\hline EA & Eurasian Patent Org. [EAPO] ${ }^{4}$ & 13.5 & complete & 23,976 & - \\
\hline GE & Georgia & 10.0 & complete & 3,693 & 2,993 \\
\hline RS & Republic of Serbia & 3.2 & complete & 4,708 & 1,923 \\
\hline GR & Greece & 29.4 & partial & 50,567 & 11,333 \\
\hline AR & Argentina & 28.7 & partial & 59,471 & 8,662 \\
\hline GT & Guatemala & 27.6 & partial & 1,010 & 212 \\
\hline IN & India & 27.4 & partial & 41,207 & 73,617 \\
\hline SG & Singapore & 26.6 & partial & 54,160 & 30,356 \\
\hline $\mathrm{CN}$ & China & 24.3 & partial & $3,587,728$ & $2,141,368$ \\
\hline EC & Ecuador & 20.0 & partial & 7,098 & 678 \\
\hline $\mathrm{CU}$ & Cuba & 19.9 & partial & 1,688 & 2,763 \\
\hline $\mathrm{PH}$ & Philippines & 19.2 & partial & 14,701 & 3,050 \\
\hline PE & Peru & 17.3 & partial & 10,752 & 1,116 \\
\hline MA & Morocco & 16.2 & partial & 11,621 & 1,728 \\
\hline IS & Iceland & 16.2 & partial & 5,481 & 3,562 \\
\hline $\mathrm{CO}$ & Colombia & 14.9 & partial & 13,458 & 3,040 \\
\hline MW & Malawi & 14.8 & partial & 428 & 18 \\
\hline $\mathrm{OA}$ & African IP Organisation [OAPI] ${ }^{5}$ & 14.7 & partial & 6,819 & - \\
\hline ZM & Zambia & 14.4 & partial & 788 & 42 \\
\hline ZW & Zimbabwe & 14.4 & partial & 2,094 & 268 \\
\hline PA & Panama & 13.6 & partial & 2,411 & 1,002 \\
\hline VN & Viet Nam & 12.8 & partial & 187 & 775 \\
\hline MT & Malta & 12.4 & partial & 126 & 516 \\
\hline TW & Chinese Taipei & 12.2 & partial & 583,999 & 566,918 \\
\hline SM & San Marino & 10.0 & partial & 190 & 210 \\
\hline SV & El Salvador & 9.8 & partial & 1,329 & 320 \\
\hline $\mathrm{KE}$ & Kenya & 9.7 & partial & 557 & 438 \\
\hline $\mathrm{MN}$ & Mongolia & 9.5 & partial & 108 & 210 \\
\hline MY & Malaysia & 9.2 & partial & 6,321 & 9,516 \\
\hline DO & Dominican Republic & 8.8 & partial & 982 & 230 \\
\hline $\mathrm{TJ}$ & Tajikistan & 8.7 & partial & 386 & 1,111 \\
\hline UA & Ukraine & 8.6 & partial & 48,114 & 54,103 \\
\hline $\mathrm{HN}$ & Honduras & 5.0 & partial & 235 & 117 \\
\hline ID & $\begin{array}{l}\text { Indonesia } \\
\text { Patent Office of the Gulf }\end{array}$ & 5.0 & partial & 14,326 & 2,146 \\
\hline GC & Coop. Council ${ }^{6}$ & 4.4 & partial & 400 & - \\
\hline $\mathrm{NI}$ & Nicaragua & 4.4 & partial & 199 & 36 \\
\hline $\mathrm{KZ}$ & Kazakhstan & 4.2 & partial & 123 & 1,790 \\
\hline $\mathrm{CL}$ & Chile & 3.8 & partial & 3,445 & 2,739 \\
\hline BA & Bosnia and Herzegovina & 3.5 & partial & 264 & 341 \\
\hline $\mathrm{DZ}$ & Algeria & 3.3 & partial & 1,391 & 586 \\
\hline $\mathrm{CR}$ & Costa Rica & 3.0 & partial & 3,443 & 814 \\
\hline UY & Uruguay & 2.2 & partial & 5,948 & 1,177 \\
\hline BY & Belarus & 0.003 & partial & 166 & 5,180 \\
\hline AM & Armenia & - & none & 55 & 548 \\
\hline$A Z$ & Azerbaijan & - & none & 51 & 1,252 \\
\hline MK & Macedonia (FYROM) & - & none & 44 & 188 \\
\hline SD & Sudan & - & none & 31 & 162 \\
\hline SY & Syrian Arab Republic & - & none & 30 & 276 \\
\hline $\mathrm{KP}$ & DPR of Korea (North) & - & none & 22 & 751 \\
\hline TN & Tunisia & - & none & 22 & 1,128 \\
\hline UZ & Uzbekistan & - & none & 15 & 1,036 \\
\hline LK & Sri Lanka & - & none & 12 & 811 \\
\hline TT & Trinidad and Tobago & - & none & 9 & 356 \\
\hline $\mathrm{GH}$ & Ghana & - & none & 6 & 230 \\
\hline KG & Kyrgyzstan & - & none & 6 & 362 \\
\hline $\mathrm{TH}$ & Thailand & - & none & 5 & 3,561 \\
\hline
\end{tabular}


The Use of Patent Statistics for International Comparisons and Analysis of Narrow Technological Fields

\begin{tabular}{|c|c|c|c|c|c|}
\hline BB & Barbados & - & none & 2 & 685 \\
\hline LR & Liberia & - & none & 2 & 28 \\
\hline$A L$ & Albania & - & none & 1 & 147 \\
\hline $\mathrm{BZ}$ & Belize & - & none & 1 & 109 \\
\hline LS & Lesotho & - & none & 1 & 10 \\
\hline LY & Libya & - & none & 1 & 34 \\
\hline VE & Venezuela & - & none & - & 2,682 \\
\hline SA & Saudi Arabia & - & none & - & 2,285 \\
\hline IR & Iran & - & none & - & 2,255 \\
\hline TM & Turkmenistan & - & none & - & 1,650 \\
\hline$A E$ & United Arab Emirates & - & none & - & 1,021 \\
\hline LB & Lebanon & - & none & - & 933 \\
\hline$B D$ & Bangladesh & - & none & - & 652 \\
\hline NG & Nigeria & - & none & - & 460 \\
\hline BO & Bolivia & - & none & - & 220 \\
\hline $\mathrm{TZ}$ & $\begin{array}{l}\text { Tanzania } \\
\text {...and other }>100 \\
\text { countries and territories }\end{array}$ & - & $\begin{array}{l}\text { none } \\
\text { none }\end{array}$ & - & 94 \\
\hline
\end{tabular}

Notes:

1 Based on TOTPAT \#7 counts (singletons + claimed priorities + duplicates)

2 Single-priority patent families, fractional counts

3 The African Regional Intellectual Property Organisation (ARIPO) has 17 member states: Botswana, Gambia, Ghana, Kenya, Lesotho, Malawi, Mozambique, Namibia, Rwanda, Sierra Leone, Somalia, Sudan, Swaziland, Uganda, Tanzania, Zambia and Zimbabwe.

4 The Eurasian Patent Organization (EAPO) has 8 member states: Turkmenistan, Belarus, Tajikistan, Russia, Azerbaijan, Armenia, Kazakhstan and Kyrgyzstan.

5 The African Intellectual Property Organisation (OAPI) has 15 member states: Benin, Burkina Faso, Cameroon, Central African Republic, Chad, Congo, Côte d'Ivoire, Gabon, Guinea, Guinea-Bissau, Mali, Mauritania, Niger, Senegal and Togo.

6 The Patent Office of the Cooperation Council for the Arab States of the Gulf (GCC) has 6 member states: United Arab Emirates, Bahrain, Saudi Arabia, Oman, Qatar and Kuwait.

This is a wealth of information from a large number of patent offices. However, given the unequal coverage across countries/offices and over time, the question is how to use this body of data for statistical analysis. Clearly, 'raw' patent counts that do not account for the unequal coverage should be interpreted with caution, particularly those with emphasis on countries and time periods for which the coverage is incomplete. Moreover, while country coverage is an important piece of information to assess patenting trends in general, it is particularly so with respect to developing countries for whom the coverage varies most.

The bias might be particularly important with respect to statistics of market protection (number of patent applications deposited at a national office). Implications for invention and co-invention indicators are less acute since some of this information can be obtained indirectly through other IP offices.

\section{Using coverage weights to generate more reliable patent statistics}

In this paper we take a step towards developing a methodology to account for differences in coverage. The detailed metadata described above can be used for this purpose and improve the reliability and representativeness of patent statistics. In particular, our intention is twofold:

i) Identify cases when one can reliably distinguish 'true' zero counts from 'missing' values. As explained above, benefits of doing so are particularly high for analyses of narrow technological fields and of countries with low levels of patenting activity (e.g. many developing countries);

ii) In cases when values are not missing, the intention is to provide an indication of reliability of the observed frequency counts. Again, this is important in data-poor environments, 
including those of narrow technological fields or countries with relatively low levels of patenting activity.

We use the PATSTAT metadata to develop coverage weights to accompany standard patent data extractions. In some sense, the weights will give the probability that the frequency counts obtained from PATSTAT reflect the 'true' count. For example, one can reasonably expect that if the coverage metadata indicate than no data batches were included in PATSTAT from patent office in country X, then the probability to have records of duplicates deposited at this country's office will be zero or very low (for singleton priorities it will indeed be zero) and for claimed priorities it will be low but nonzero. This approach will allow choosing the minimum level of coverage that is desired or appropriate for a given application (i.e. it allows sample selection).

First, we summarize the information provided by the EPO in the "contents and coverage of DOCDB bibliographic files" and construct a statistic that represents the fraction of a given year for which PATSTAT has batches of data from a given patent office, ranging between "0" (office $i$ in year $t$ not covered at all) and " 1 " (full coverage) (e.g. if data is available only for 3 months of a year ten weight $=0.25$ ). Table 3 gives the weights for offices with at least a partial coverage. 
The Use of Patent Statistics for International Comparisons and Analysis of Narrow Technological Fields

Table 3. Coverage weights of patent offices in PATSTAT APR12, 1980-2009

\begin{tabular}{|c|c|c|c|c|c|c|c|c|c|c|c|c|c|c|c|c|}
\hline & 1980 & 1981 & 1982 & 1983 & 1984 & 1985 & 1986 & 1987 & 1988 & 1989 & 1990 & 1991 & 1992 & 1993 & 1994 & 1995 \\
\hline AP & 0 & 0 & 0 & 0 & 0.167 & 1 & 1 & 1 & 1 & 1 & 1 & 1 & 1 & 1 & 1 & 1 \\
\hline AR & 1 & 1 & 1 & 1 & 1 & 1 & 1 & 1 & 1 & 1 & 1 & 1 & 1 & 1 & 1 & 0.630 \\
\hline AT & 1 & 1 & 1 & 1 & 1 & 1 & 1 & 1 & 1 & 1 & 1 & 1 & 1 & 1 & 1 & 1 \\
\hline $\mathrm{AU}$ & 1 & 1 & 1 & 1 & 1 & 1 & 1 & 1 & 1 & 1 & 1 & 1 & 1 & 1 & 1 & 1 \\
\hline BA & 0 & 0 & 0 & 0 & 0 & 0 & 0 & 0 & 0 & 0 & 0 & 0 & 0 & 0 & 0 & 0 \\
\hline $\mathrm{BE}$ & 1 & 1 & 1 & 1 & 1 & 1 & 1 & 1 & 1 & 1 & 1 & 1 & 1 & 1 & 1 & 1 \\
\hline BG & 1 & 1 & 1 & 1 & 1 & 1 & 1 & 1 & 1 & 1 & 1 & 1 & 1 & 1 & 1 & 1 \\
\hline $\mathrm{BR}$ & 1 & 1 & 1 & 1 & 1 & 1 & 1 & 1 & 1 & 1 & 1 & 1 & 1 & 1 & 1 & 1 \\
\hline BY & 0 & 0 & 0 & 0 & 0 & 0 & 0 & 0 & 0 & 0 & 0 & 0 & 0 & 0 & 0 & 0 \\
\hline CA & 1 & 1 & 1 & 1 & 1 & 1 & 1 & 1 & 1 & 1 & 1 & 1 & 1 & 1 & 1 & 1 \\
\hline $\mathrm{CH}$ & 1 & 1 & 1 & 1 & 1 & 1 & 1 & 1 & 1 & 1 & 1 & 1 & 1 & 1 & 1 & 1 \\
\hline$C L$ & 0 & 0 & 0 & 0 & 0 & 0 & 0 & 0 & 0 & 0 & 0 & 0 & 0 & 0 & 0 & 0 \\
\hline $\mathrm{CN}$ & 0 & 0 & 0 & 0 & 0 & 0.310 & 1 & 1 & 0.992 & 0.992 & 1 & 1 & 1 & 1 & 1 & 1 \\
\hline $\mathrm{CO}$ & 0 & 0 & 0 & 0 & 0 & 0 & 0 & 0 & 0 & 0 & 0 & 0 & 0 & 0 & 0 & 0.882 \\
\hline $\mathrm{CR}$ & 0 & 0 & 0 & 0 & 0 & 0 & 0 & 0 & 0 & 0 & 0 & 0 & 0 & 0 & 0 & 0 \\
\hline CS & 1 & 1 & 1 & 1 & 1 & 1 & 1 & 1 & 1 & 1 & 1 & 1 & 1 & 0.956 & 0 & 0 \\
\hline $\mathrm{CU}$ & 1 & 1 & 1 & 1 & 1 & 1 & 1 & 1 & 1 & 1 & 1 & 1 & 1 & 1 & 1 & 0.945 \\
\hline $\mathrm{CY}$ & 1 & 1 & 1 & 1 & 1 & 1 & 1 & 1 & 1 & 1 & 1 & 1 & 1 & 1 & 1 & 1 \\
\hline $\mathrm{CZ}$ & 0 & 0 & 0 & 0 & 0 & 0 & 0 & 0 & 0 & 0 & 0 & 0 & 0 & 0.795 & 1 & 1 \\
\hline $\mathrm{DD}$ & 1 & 1 & 1 & 1 & 1 & 1 & 1 & 1 & 1 & 1 & 1 & 1 & 1 & 1 & 1 & 1 \\
\hline $\mathrm{DE}$ & 1 & 1 & 1 & 1 & 1 & 1 & 1 & 1 & 1 & 1 & 1 & 1 & 1 & 1 & 1 & 1 \\
\hline DK & 1 & 1 & 1 & 1 & 1 & 1 & 1 & 1 & 1 & 1 & 1 & 1 & 1 & 1 & 1 & 1 \\
\hline DO & 0 & 0 & 0 & 0 & 0 & 0 & 0 & 0 & 0 & 0 & 0 & 0 & 0 & 0 & 0 & 0 \\
\hline $\mathrm{DZ}$ & 0 & 0 & 0 & 0 & 0 & 0 & 0 & 0 & 0 & 0 & 0 & 0 & 0 & 0 & 0 & 0 \\
\hline EA & 0 & 0 & 0 & 0 & 0 & 0 & 0 & 0 & 0 & 0 & 0 & 0 & 0 & 0 & 0 & 0 \\
\hline EC & 0 & 0 & 0 & 0 & 0 & 0 & 0 & 0 & 0 & 0 & 1 & 1 & 1 & 1 & 1 & 1 \\
\hline $\mathrm{EE}$ & 0 & 0 & 0 & 0 & 0 & 0 & 0 & 0 & 0 & 0 & 0 & 0 & 0 & 0 & 0 & 0.047 \\
\hline EG & 1 & 1 & 1 & 1 & 1 & 1 & 1 & 1 & 1 & 1 & 1 & 1 & 1 & 1 & 1 & 1 \\
\hline EP & 1 & 1 & 1 & 1 & 1 & 1 & 1 & 1 & 1 & 1 & 1 & 1 & 1 & 1 & 1 & 1 \\
\hline ES & 1 & 1 & 1 & 1 & 1 & 1 & 1 & 1 & 1 & 1 & 1 & 1 & 1 & 1 & 1 & 1 \\
\hline $\mathrm{FI}$ & 1 & 1 & 1 & 1 & 1 & 1 & 1 & 1 & 1 & 1 & 1 & 1 & 1 & 1 & 1 & 1 \\
\hline FR & 1 & 1 & 1 & 1 & 1 & 1 & 1 & 1 & 1 & 1 & 1 & 1 & 1 & 1 & 1 & 1 \\
\hline GB & 1 & 1 & 1 & 1 & 1 & 1 & 1 & 1 & 1 & 1 & 1 & 1 & 1 & 1 & 1 & 1 \\
\hline GC & 0 & 0 & 0 & 0 & 0 & 0 & 0 & 0 & 0 & 0 & 0 & 0 & 0 & 0 & 0 & 0 \\
\hline GE & 0 & 0 & 0 & 0 & 0 & 0 & 0 & 0 & 0 & 0 & 0 & 0 & 0 & 0 & 0 & 0 \\
\hline GR & 1 & 1 & 1 & 1 & 1 & 1 & 1 & 1 & 0.352 & 1 & 1 & 1 & 1 & 1 & 1 & 1 \\
\hline GT & 1 & 1 & 1 & 1 & 1 & 1 & 1 & 1 & 1 & 1 & 1 & 1 & 1 & 1 & 1 & 1 \\
\hline HK & 1 & 1 & 1 & 1 & 1 & 1 & 1 & 1 & 1 & 1 & 1 & 1 & 1 & 1 & 1 & 1 \\
\hline HN & 0 & 0 & 0 & 0 & 0 & 0 & 0 & 0 & 0 & 0 & 0 & 0 & 0 & 0 & 0 & 0 \\
\hline $\mathrm{HR}$ & 0 & 0 & 0 & 0 & 0 & 0 & 0 & 0 & 0 & 0 & 0 & 0 & 0 & 0 & 0.392 & 1 \\
\hline $\mathrm{HU}$ & 1 & 1 & 1 & 1 & 1 & 1 & 1 & 1 & 1 & 1 & 1 & 1 & 1 & 1 & 1 & 1 \\
\hline ID & 0 & 0 & 0 & 0 & 0 & 0 & 0 & 0 & 0 & 0 & 0 & 0 & 0 & 0 & 0 & 0 \\
\hline IE & 1 & 1 & 1 & 1 & 1 & 1 & 1 & 1 & 1 & 1 & 1 & 1 & 1 & 1 & 1 & 1 \\
\hline IL & 1 & 1 & 1 & 1 & 1 & 1 & 1 & 1 & 1 & 1 & 1 & 1 & 1 & 1 & 1 & 1 \\
\hline IN & 1 & 1 & 1 & 1 & 1 & 1 & 1 & 1 & 1 & 1 & 1 & 1 & 1 & 1 & 1 & 1 \\
\hline IS & 0 & 0 & 0 & 0 & 0 & 0 & 0 & 0 & 0 & 0 & 0 & 0 & 0 & 0.189 & 1 & 1 \\
\hline IT & 1 & 1 & 1 & 1 & 1 & 1 & 1 & 1 & 1 & 1 & 1 & 1 & 1 & 1 & 1 & 1 \\
\hline
\end{tabular}


Table 3. (cont.)

\begin{tabular}{|c|c|c|c|c|c|c|c|c|c|c|c|c|c|c|c|c|}
\hline & 1980 & 1981 & 1982 & 1983 & 1984 & 1985 & 1986 & 1987 & 1988 & 1989 & 1990 & 1991 & 1992 & 1993 & 1994 & 1995 \\
\hline $\mathrm{JP}$ & 1 & 1 & 1 & 1 & 1 & 1 & 1 & 1 & 1 & 1 & 1 & 1 & 1 & 1 & 1 & 1 \\
\hline $\mathrm{KE}$ & 1 & 1 & 1 & 1 & 1 & 1 & 1 & 1 & 1 & 0.668 & 0 & 0 & 0 & 0 & 0 & 0 \\
\hline $\mathrm{KR}$ & 1 & 1 & 1 & 1 & 1 & 1 & 1 & 1 & 1 & 1 & 1 & 1 & 1 & 1 & 1 & 1 \\
\hline KZ & 0 & 0 & 0 & 0 & 0 & 0 & 0 & 0 & 0 & 0 & 0 & 0 & 0 & 0 & 0 & 0 \\
\hline LT & 0 & 0 & 0 & 0 & 0 & 0 & 0 & 0 & 0 & 0 & 0 & 0 & 0.213 & 1 & 1 & 1 \\
\hline LU & 1 & 1 & 1 & 1 & 1 & 1 & 1 & 1 & 1 & 1 & 1 & 1 & 1 & 1 & 1 & 1 \\
\hline LV & 0 & 0 & 0 & 0 & 0 & 0 & 0 & 0 & 0 & 0 & 0 & 0 & 0 & 0 & 0.814 & 1 \\
\hline MA & 0 & 0 & 0 & 0 & 0 & 0 & 0 & 0 & 0 & 0 & 0 & 0 & 0 & 0.197 & 1 & 1 \\
\hline$M C$ & 1 & 1 & 1 & 1 & 1 & 1 & 1 & 1 & 1 & 1 & 1 & 1 & 1 & 1 & 1 & 1 \\
\hline MD & 0 & 0 & 0 & 0 & 0 & 0 & 0 & 0 & 0 & 0 & 0 & 0 & 0 & 0 & 0.589 & 1 \\
\hline MN & 1 & 1 & 1 & 1 & 1 & 1 & 1 & 1 & 1 & 0.455 & 0 & 0 & 0 & 0 & 0 & 0 \\
\hline MT & 1 & 1 & 1 & 1 & 1 & 1 & 1 & 1 & 1 & 1 & 1 & 1 & 0.352 & 0 & 0 & 0 \\
\hline MW & 1 & 1 & 1 & 1 & 1 & 1 & 1 & 1 & 1 & 1 & 1 & 1 & 1 & 1 & 0.781 & 0 \\
\hline$M X$ & 0.997 & 1 & 1 & 1 & 1 & 1 & 1 & 1 & 1 & 1 & 1 & 1 & 1 & 1 & 1 & 1 \\
\hline MY & 1 & 1 & 1 & 1 & 1 & 1 & 1 & 1 & 1 & 0.205 & 0 & 0 & 0 & 0 & 0 & 0 \\
\hline $\mathrm{NI}$ & 0 & 0 & 0 & 0 & 0 & 0 & 0 & 0 & 0 & 0 & 0 & 0 & 0 & 0 & 0 & 0 \\
\hline $\mathrm{NL}$ & 1 & 1 & 1 & 1 & 1 & 1 & 1 & 1 & 1 & 1 & 1 & 1 & 1 & 1 & 1 & 1 \\
\hline NO & 1 & 1 & 1 & 1 & 1 & 1 & 1 & 1 & 1 & 1 & 1 & 1 & 1 & 1 & 1 & 1 \\
\hline NZ & 1 & 1 & 1 & 1 & 1 & 1 & 1 & 1 & 1 & 1 & 1 & 1 & 1 & 1 & 1 & 1 \\
\hline$O A$ & 0 & 0 & 0 & 0 & 0 & 0 & 0 & 0 & 0 & 0 & 0 & 0 & 0.380 & 1 & 1 & 1 \\
\hline PA & 0 & 0 & 0 & 0 & 0 & 0 & 0 & 0 & 0 & 0 & 0 & 0 & 0 & 0 & 0 & 0 \\
\hline $\mathrm{PE}$ & 0 & 0 & 0 & 0 & 0 & 0 & 0 & 0 & 0 & 0 & 0 & 0 & 0.254 & 1 & 1 & 1 \\
\hline $\mathrm{PH}$ & 1 & 1 & 1 & 1 & 1 & 1 & 1 & 1 & 1 & 1 & 1 & 1 & 1 & 1 & 1 & 1 \\
\hline $\mathrm{PL}$ & 1 & 1 & 1 & 1 & 1 & 1 & 1 & 1 & 1 & 1 & 1 & 1 & 1 & 1 & 1 & 1 \\
\hline PT & 1 & 1 & 1 & 1 & 1 & 1 & 1 & 1 & 1 & 1 & 1 & 1 & 1 & 1 & 1 & 1 \\
\hline RO & 1 & 1 & 1 & 1 & 1 & 1 & 1 & 1 & 1 & 1 & 1 & 1 & 1 & 1 & 1 & 1 \\
\hline RS & 0 & 0 & 0 & 0 & 0 & 0 & 0 & 0 & 0 & 0 & 0 & 0 & 0 & 0 & 0 & 0 \\
\hline $\mathrm{RU}$ & 0 & 0 & 0 & 0 & 0 & 0 & 0 & 0 & 0 & 0 & 0 & 0 & 0 & 0.877 & 1 & 1 \\
\hline SE & 1 & 1 & 1 & 1 & 1 & 1 & 1 & 1 & 1 & 1 & 1 & 1 & 1 & 1 & 1 & 1 \\
\hline SG & 0 & 0 & 0 & 0.849 & 1 & 1 & 1 & 1 & 1 & 1 & 1 & 1 & 1 & 1 & 1 & 0.762 \\
\hline $\mathrm{SI}$ & 0 & 0 & 0 & 0 & 0 & 0 & 0 & 0 & 0 & 0 & 0 & 0 & 0.096 & 1 & 1 & 1 \\
\hline SK & 0 & 0 & 0 & 0 & 0 & 0 & 0 & 0 & 0 & 0 & 0 & 0 & 0 & 0.167 & 1 & 1 \\
\hline SM & 0 & 0 & 0 & 0 & 0 & 0 & 0 & 0 & 0 & 0 & 0 & 0 & 0 & 0 & 0 & 0 \\
\hline SU & 1 & 1 & 1 & 1 & 1 & 1 & 1 & 1 & 1 & 1 & 1 & 1 & 1 & 1 & 1 & 1 \\
\hline SV & 0 & 0 & 0 & 0 & 0 & 0 & 0 & 0 & 0 & 0 & 0 & 0 & 0 & 0 & 0 & 0 \\
\hline $\mathrm{TJ}$ & 0 & 0 & 0 & 0 & 0 & 0 & 0 & 0 & 0 & 0 & 0 & 0 & 0 & 0 & 0 & 0 \\
\hline TR & 1 & 1 & 1 & 1 & 1 & 1 & 1 & 1 & 1 & 1 & 1 & 1 & 1 & 1 & 1 & 1 \\
\hline TW & 0 & 0 & 0 & 0 & 0 & 0 & 0 & 0 & 0 & 0 & 0 & 0 & 0 & 0 & 0 & 0 \\
\hline UA & 0 & 0 & 0 & 0 & 0 & 0 & 0 & 0 & 0 & 0 & 0 & 0 & 0 & 0 & 0 & 0 \\
\hline US & 1 & 1 & 1 & 1 & 1 & 1 & 1 & 1 & 1 & 1 & 1 & 1 & 1 & 1 & 1 & 1 \\
\hline UY & 0 & 0 & 0 & 0 & 0 & 0 & 0 & 0 & 0 & 0 & 0 & 0 & 0 & 0 & 0 & 0 \\
\hline VN & 0 & 0 & 0 & 0 & 0.489 & 1 & 1 & 1 & 1 & 1 & 1 & 1 & 1 & 1 & 1 & 1 \\
\hline wo & 1 & 1 & 1 & 1 & 1 & 1 & 1 & 1 & 1 & 1 & 1 & 1 & 1 & 1 & 1 & 1 \\
\hline YU & 1 & 1 & 1 & 1 & 1 & 1 & 1 & 1 & 1 & 1 & 1 & 1 & 0.407 & 0 & 0 & 0 \\
\hline $\mathrm{ZA}$ & 1 & 1 & 1 & 1 & 1 & 1 & 1 & 1 & 1 & 1 & 1 & 1 & 1 & 1 & 1 & 1 \\
\hline ZM & 1 & 1 & 1 & 1 & 1 & 1 & 1 & 1 & 1 & 1 & 1 & 1 & 1 & 1 & 0.397 & 0 \\
\hline ZW & 0.328 & 1 & 1 & 1 & 1 & 1 & 1 & 1 & 1 & 1 & 1 & 1 & 1 & 1 & 1 & 0.068 \\
\hline
\end{tabular}


Table 3. (cont.)

\begin{tabular}{|c|c|c|c|c|c|c|c|c|c|c|c|c|c|c|c|}
\hline & 1996 & 1997 & 1998 & 1999 & 2000 & 2001 & 2002 & 2003 & 2004 & 2005 & 2006 & 2007 & 2008 & 2009 & $1980-2009$ \\
\hline AP & 1 & 1 & 1 & 1 & 1 & 1 & 1 & 1 & 1 & 1 & 1 & 1 & 1 & 1 & 25.167 \\
\hline AR & 0.077 & 1 & 1 & 1 & 1 & 1 & 1 & 1 & 1 & 1 & 1 & 1 & 1 & 1 & 28.707 \\
\hline AT & 1 & 1 & 1 & 1 & 1 & 1 & 1 & 1 & 1 & 1 & 1 & 1 & 1 & 1 & 30.000 \\
\hline $\mathrm{AU}$ & 1 & 1 & 1 & 1 & 1 & 1 & 1 & 1 & 1 & 1 & 1 & 1 & 1 & 1 & 30.000 \\
\hline BA & 0 & 0 & 0.825 & 1 & 1 & 0.704 & 0 & 0 & 0 & 0 & 0 & 0 & 0 & 0 & 3.529 \\
\hline BE & 1 & 1 & 1 & 1 & 1 & 1 & 1 & 1 & 1 & 1 & 1 & 1 & 1 & 1 & 30.000 \\
\hline BG & 1 & 1 & 1 & 1 & 1 & 1 & 1 & 1 & 1 & 1 & 1 & 1 & 1 & 1 & 30.000 \\
\hline BR & 1 & 1 & 1 & 1 & 1 & 1 & 1 & 1 & 1 & 1 & 1 & 1 & 1 & 1 & 30.000 \\
\hline BY & 0 & 0.003 & 0 & 0 & 0 & 0 & 0 & 0 & 0 & 0 & 0 & 0 & 0 & 0 & 0.003 \\
\hline $\mathrm{CA}$ & 1 & 1 & 1 & 1 & 1 & 1 & 1 & 1 & 1 & 1 & 1 & 1 & 1 & 1 & 30.000 \\
\hline $\mathrm{CH}$ & 1 & 1 & 1 & 1 & 1 & 1 & 1 & 1 & 1 & 1 & 1 & 1 & 1 & 1 & 30.000 \\
\hline $\mathrm{CL}$ & 0 & 0 & 0 & 0 & 0 & 0 & 0 & 0 & 0 & 0.984 & 1 & 1 & 0.814 & 0 & 3.798 \\
\hline $\mathrm{CN}$ & 1 & 1 & 1 & 1 & 1 & 1 & 1 & 1 & 1 & 1 & 1 & 1 & 1 & 1 & 24.293 \\
\hline $\mathrm{CO}$ & 1 & 1 & 1 & 1 & 1 & 1 & 1 & 1 & 1 & 1 & 1 & 1 & 1 & 1 & 14.882 \\
\hline $\mathrm{CR}$ & 0 & 0 & 0 & 0 & 0 & 0 & 0 & 0 & 0 & 0 & 0 & 0.992 & 1 & 1 & 2.992 \\
\hline CS & 0 & 0 & 0 & 0 & 0 & 0 & 0 & 0 & 0 & 0 & 0 & 0 & 0 & 0 & 13.956 \\
\hline $\mathrm{CU}$ & 0 & 0 & 0 & 0 & 0 & 0 & 0 & 0 & 0 & 0 & 0.948 & 1 & 1 & 1 & 19.893 \\
\hline $\mathrm{CY}$ & 1 & 1 & 1 & 1 & 1 & 1 & 1 & 1 & 1 & 1 & 1 & 1 & 1 & 1 & 30.000 \\
\hline $\mathrm{CZ}$ & 1 & 1 & 1 & 1 & 1 & 1 & 1 & 1 & 1 & 1 & 1 & 1 & 1 & 1 & 16.795 \\
\hline $\mathrm{DD}$ & 1 & 1 & 1 & 0.537 & 0 & 0 & 0 & 0 & 0 & 0 & 0 & 0 & 0 & 0 & 19.537 \\
\hline $\mathrm{DE}$ & 1 & 1 & 1 & 1 & 1 & 1 & 1 & 1 & 1 & 1 & 1 & 1 & 1 & 1 & 30.000 \\
\hline DK & 1 & 1 & 1 & 1 & 1 & 1 & 1 & 1 & 1 & 1 & 1 & 1 & 1 & 1 & 30.000 \\
\hline DO & 0 & 0 & 0 & 0 & 0 & 0.756 & 1 & 1 & 1 & 1 & 1 & 1 & 1 & 1 & 8.756 \\
\hline $\mathrm{DZ}$ & 0 & 0 & 0 & 0 & 0 & 0 & 0.934 & 1 & 1 & 0.408 & 0 & 0 & 0 & 0 & 3.342 \\
\hline EA & 0.503 & 1 & 1 & 1 & 1 & 1 & 1 & 1 & 1 & 1 & 1 & 1 & 1 & 1 & 13.503 \\
\hline EC & 1 & 1 & 1 & 1 & 1 & 1 & 1 & 1 & 1 & 1 & 1 & 1 & 1 & 1 & 20.000 \\
\hline EE & 1 & 1 & 1 & 1 & 1 & 1 & 1 & 1 & 1 & 1 & 1 & 1 & 1 & 1 & 14.047 \\
\hline EG & 1 & 1 & 1 & 1 & 1 & 1 & 1 & 1 & 1 & 1 & 1 & 1 & 1 & 1 & 30.000 \\
\hline EP & 1 & 1 & 1 & 1 & 1 & 1 & 1 & 1 & 1 & 1 & 1 & 1 & 1 & 1 & 30.000 \\
\hline ES & 1 & 1 & 1 & 1 & 1 & 1 & 1 & 1 & 1 & 1 & 1 & 1 & 1 & 1 & 30.000 \\
\hline $\mathrm{FI}$ & 1 & 1 & 1 & 1 & 1 & 1 & 1 & 1 & 1 & 1 & 1 & 1 & 1 & 1 & 30.000 \\
\hline FR & 1 & 1 & 1 & 1 & 1 & 1 & 1 & 1 & 1 & 1 & 1 & 1 & 1 & 1 & 30.000 \\
\hline GB & 1 & 1 & 1 & 1 & 1 & 1 & 1 & 1 & 1 & 1 & 1 & 1 & 1 & 1 & 30.000 \\
\hline GC & 0 & 0 & 0 & 0 & 0 & 0 & 0.173 & 1 & 1 & 1 & 1 & 0.247 & 0 & 0 & 4.419 \\
\hline GE & 0 & 0 & 0 & 0 & 0.975 & 1 & 1 & 1 & 1 & 1 & 1 & 1 & 1 & 1 & 9.975 \\
\hline GR & 1 & 1 & 1 & 1 & 1 & 1 & 1 & 1 & 1 & 1 & 1 & 1 & 1 & 1 & 29.352 \\
\hline GT & 1 & 1 & 1 & 1 & 1 & 1 & 1 & 1 & 1 & 1 & 1 & 0.636 & 0 & 0 & 27.636 \\
\hline HK & 1 & 1 & 1 & 1 & 1 & 1 & 1 & 1 & 1 & 1 & 1 & 1 & 1 & 1 & 30.000 \\
\hline $\mathrm{HN}$ & 0 & 0 & 0 & 0 & 0 & 0 & 0 & 0 & 0 & 0.967 & 1 & 1 & 1 & 1 & 4.967 \\
\hline HR & 1 & 1 & 1 & 1 & 1 & 1 & 1 & 1 & 1 & 1 & 1 & 1 & 1 & 1 & 15.392 \\
\hline $\mathrm{HU}$ & 1 & 1 & 1 & 1 & 1 & 1 & 1 & 1 & 1 & 1 & 1 & 1 & 1 & 1 & 30.000 \\
\hline ID & 0.478 & 0.479 & 1 & 1 & 1 & 1 & 0.008 & 0 & 0 & 0 & 0 & 0 & 0 & 0 & 4.966 \\
\hline $\mathrm{IE}$ & 1 & 1 & 1 & 1 & 1 & 1 & 1 & 1 & 1 & 1 & 1 & 1 & 1 & 1 & 30.000 \\
\hline IL & 1 & 1 & 1 & 1 & 1 & 1 & 1 & 1 & 1 & 1 & 1 & 1 & 1 & 1 & 30.000 \\
\hline IN & 1 & 1 & 1 & 1 & 1 & 1 & 1 & 1 & 1 & 1 & 1 & 0.359 & 0 & 0 & 27.359 \\
\hline IS & 1 & 1 & 1 & 1 & 1 & 1 & 1 & 1 & 1 & 1 & 1 & 1 & 1 & 1 & 16.189 \\
\hline $\mathrm{IT}$ & 1 & 1 & 1 & 1 & 1 & 1 & 1 & 1 & 1 & 1 & 1 & 1 & 1 & 1 & 30.000 \\
\hline
\end{tabular}


Table 3. (cont.)

\begin{tabular}{|c|c|c|c|c|c|c|c|c|c|c|c|c|c|c|c|}
\hline & 1996 & 1997 & 1998 & 1999 & 2000 & 2001 & 2002 & 2003 & 2004 & 2005 & 2006 & 2007 & 2008 & 2009 & $1980-2009$ \\
\hline $\mathrm{JP}$ & 1 & 1 & 1 & 1 & 1 & 1 & 1 & 1 & 1 & 1 & 1 & 1 & 1 & 1 & 30.000 \\
\hline KE & 0 & 0 & 0 & 0 & 0 & 0 & 0 & 0 & 0 & 0 & 0 & 0 & 0 & 0 & 9.668 \\
\hline $\mathrm{KR}$ & 1 & 1 & 1 & 1 & 1 & 1 & 1 & 1 & 1 & 1 & 1 & 1 & 1 & 1 & 30.000 \\
\hline KZ & 0 & 0 & 0 & 0 & 0 & 0 & 0 & 0 & 0.874 & 1 & 1 & 1 & 0.372 & 0 & 4.246 \\
\hline LT & 1 & 1 & 1 & 1 & 1 & 1 & 1 & 1 & 1 & 1 & 1 & 1 & 1 & 1 & 17.213 \\
\hline LU & 1 & 1 & 1 & 1 & 1 & 1 & 1 & 1 & 1 & 1 & 1 & 1 & 1 & 1 & 30.000 \\
\hline LV & 1 & 1 & 1 & 1 & 1 & 1 & 1 & 1 & 1 & 1 & 1 & 1 & 1 & 1 & 15.814 \\
\hline MA & 1 & 1 & 1 & 1 & 1 & 1 & 1 & 1 & 1 & 1 & 1 & 1 & 1 & 1 & 16.197 \\
\hline$M C$ & 1 & 1 & 1 & 1 & 1 & 1 & 1 & 1 & 1 & 1 & 1 & 1 & 1 & 1 & 30.000 \\
\hline$M D$ & 1 & 1 & 1 & 1 & 1 & 1 & 1 & 1 & 1 & 1 & 1 & 1 & 1 & 1 & 15.589 \\
\hline MN & 0 & 0 & 0 & 0 & 0 & 0 & 0 & 0 & 0 & 0 & 0 & 0 & 0 & 0 & 9.455 \\
\hline MT & 0 & 0 & 0 & 0 & 0 & 0 & 0 & 0 & 0 & 0 & 0 & 0 & 0 & 0 & 12.352 \\
\hline MW & 0 & 0 & 0 & 0 & 0 & 0 & 0 & 0 & 0 & 0 & 0 & 0 & 0 & 0 & 14.781 \\
\hline$M X$ & 1 & 1 & 1 & 1 & 1 & 1 & 1 & 1 & 1 & 1 & 1 & 1 & 1 & 1 & 29.997 \\
\hline MY & 0 & 0 & 0 & 0 & 0 & 0 & 0 & 0 & 0 & 0 & 0 & 0 & 0 & 0 & 9.205 \\
\hline $\mathrm{NI}$ & 0 & 0 & 0 & 0 & 0 & 0 & 0 & 0.156 & 1 & 1 & 1 & 1 & 0.230 & 0 & 4.386 \\
\hline $\mathrm{NL}$ & 1 & 1 & 1 & 1 & 1 & 1 & 1 & 1 & 1 & 1 & 1 & 1 & 1 & 1 & 30.000 \\
\hline NO & 1 & 1 & 1 & 1 & 1 & 1 & 1 & 1 & 1 & 1 & 1 & 1 & 1 & 1 & 30.000 \\
\hline $\mathrm{NZ}$ & 1 & 1 & 1 & 1 & 1 & 1 & 1 & 1 & 1 & 1 & 1 & 1 & 1 & 1 & 30.000 \\
\hline $\mathrm{OA}$ & 1 & 1 & 1 & 1 & 1 & 1 & 1 & 1 & 1 & 1 & 1 & 0.282 & 0 & 0 & 14.662 \\
\hline PA & 0.617 & 1 & 1 & 1 & 1 & 1 & 1 & 1 & 1 & 1 & 1 & 1 & 1 & 1 & 13.617 \\
\hline $\mathrm{PE}$ & 1 & 1 & 1 & 1 & 1 & 1 & 1 & 1 & 1 & 1 & 1 & 1 & 1 & 1 & 17.254 \\
\hline PH & 1 & 1 & 1 & 0.167 & 0 & 0 & 0 & 0 & 0 & 0 & 0 & 0 & 0 & 0 & 19.167 \\
\hline $\mathrm{PL}$ & 1 & 1 & 1 & 1 & 1 & 1 & 1 & 1 & 1 & 1 & 1 & 1 & 1 & 1 & 30.000 \\
\hline PT & 1 & 1 & 1 & 1 & 1 & 1 & 1 & 1 & 1 & 1 & 1 & 1 & 1 & 1 & 30.000 \\
\hline RO & 1 & 1 & 1 & 1 & 1 & 1 & 1 & 1 & 1 & 1 & 1 & 1 & 1 & 1 & 30.000 \\
\hline RS & 0 & 0 & 0 & 0 & 0 & 0 & 0 & 0 & 0 & 0 & 0.181 & 1 & 1 & 1 & 3.181 \\
\hline $\mathrm{RU}$ & 1 & 1 & 1 & 1 & 1 & 1 & 1 & 1 & 1 & 1 & 1 & 1 & 1 & 1 & 16.877 \\
\hline SE & 1 & 1 & 1 & 1 & 1 & 1 & 1 & 1 & 1 & 1 & 1 & 1 & 1 & 1 & 30.000 \\
\hline SG & 1 & 1 & 1 & 1 & 1 & 1 & 1 & 1 & 1 & 1 & 1 & 1 & 1 & 1 & 26.611 \\
\hline $\mathrm{SI}$ & 1 & 1 & 1 & 1 & 1 & 1 & 1 & 1 & 1 & 1 & 1 & 1 & 1 & 1 & 17.096 \\
\hline SK & 1 & 1 & 1 & 1 & 1 & 1 & 1 & 1 & 1 & 1 & 1 & 1 & 1 & 1 & 16.167 \\
\hline SM & 0 & 0 & 0 & 0 & 1 & 1 & 1 & 1 & 1 & 1 & 1 & 1 & 1 & 1 & 10.000 \\
\hline SU & 1 & 1 & 1 & 1 & 1 & 1 & 1 & 1 & 1 & 1 & 1 & 1 & 1 & 1 & 30.000 \\
\hline SV & 0 & 0 & 0 & 0 & 0.801 & 1 & 1 & 1 & 1 & 1 & 1 & 1 & 1 & 1 & 9.801 \\
\hline $\mathrm{TJ}$ & 0 & 0 & 0.132 & 1 & 1 & 1 & 1 & 1 & 1 & 1 & 1 & 0.540 & 0 & 0 & 8.671 \\
\hline TR & 1 & 1 & 1 & 1 & 1 & 1 & 1 & 1 & 1 & 1 & 1 & 1 & 1 & 1 & 30.000 \\
\hline TW & 0 & 0.167 & 1 & 1 & 1 & 1 & 1 & 1 & 1 & 1 & 1 & 1 & 1 & 1 & 12.167 \\
\hline UA & 0 & 0 & 0 & 0.225 & 1 & 1 & 1 & 1 & 1 & 1 & 1 & 1 & 0.402 & 0 & 8.626 \\
\hline US & 1 & 1 & 1 & 1 & 1 & 1 & 1 & 1 & 1 & 1 & 1 & 1 & 1 & 1 & 30.000 \\
\hline UY & 0 & 0 & 0 & 0 & 0 & 0 & 0 & 0 & 0 & 0 & 0 & 0.170 & 1 & 1 & 2.170 \\
\hline VN & 1 & 0.315 & 0 & 0 & 0 & 0 & 0 & 0 & 0 & 0 & 0 & 0 & 0 & 0 & 12.804 \\
\hline wo & 1 & 1 & 1 & 1 & 1 & 1 & 1 & 1 & 1 & 1 & 1 & 1 & 1 & 1 & 30.000 \\
\hline YU & 0.981 & 1 & 1 & 1 & 1 & 1 & 1 & 1 & 1 & 1 & 0.956 & 0 & 0 & 0 & 23.344 \\
\hline $\mathrm{ZA}$ & 1 & 1 & 1 & 1 & 1 & 1 & 1 & 1 & 1 & 1 & 1 & 1 & 1 & 1 & 30.000 \\
\hline ZM & 0 & 0 & 0 & 0 & 0 & 0 & 0 & 0 & 0 & 0 & 0 & 0 & 0 & 0 & 14.397 \\
\hline ZW & 0 & 0 & 0 & 0 & 0 & 0 & 0 & 0 & 0 & 0 & 0 & 0 & 0 & 0 & 14.396 \\
\hline
\end{tabular}


Second, we define a set of rules to assign these "coverage weights" to different types of patent documents extracted from PATSTAT (Table 4). On the one hand, any information derived from a singleton patent application will be assigned a weight corresponding to the coverage of its priority application office. The weight is equal to the theoretical coverage of the priority application office in the application year. On the other hand, information derived from a family of patent applications (claimed priorities) is assigned a weight corresponding to maximum weight associated with the priority and/or duplicate application offices of corresponding family members. And finally, information derived from duplicate patent applications due to absence of data from the priority office is assigned a weight corresponding to the duplicate application office.

Table 4. Assignment rules for coverage weights

\begin{tabular}{ll}
\hline Document type & Coverage weight \\
\hline Singleton priority & $w^{P R I O}$ \\
Claimed priority & $\max \left(w^{P R I O}, w^{P U P L}\right)$ \\
Duplicate application & $w^{D U P L}$ \\
\hline
\end{tabular}

The unequal country coverage has only a limited effect on our ability to identify inventions that have sought protection elsewhere ('claimed priorities'), because we can impute inventor information from other members of the same patent family whose coverage might be superior. However, it does potentially compromise our ability to identify inventions that have sought protection only in one jurisdiction (singletons) with incomplete coverage, or duplicate applications of foreign patents (duplicates) registered in jurisdictions with incomplete coverage.

It must be noted that the approach presented here has certain limitations: First, the coverage weights as derived here represent only the direct coverage of countries in PATSTAT. Indirect coverage, derived by exploiting information on patent families, is not reflected (see e.g. the lower part of Table 2). Second, the coverage weights are only assigned to patent offices, not inventor countries (this would help with interpretation of the last column in Table 2). In both cases, addressing these issues would require estimating such propensities econometrically. However, this would require access to data external to PATSTAT.

Third, we generate frequency counts along selected vectors - by office, inventor country, priority or application year, technology, as well as the coverage weight. This allows making judgements about the reliability of observed patent counts, notably to:

- Identify 'true zeros' - i.e. when patent count=0 and coverage weight=1 (or close to 1 ). Such distinction between 'true zeros' and 'missing values is otherwise not possible.

- Identify nonzero counts with 'low reliability' - i.e. when patent count $>0$ and weight $<1$ (or far from 1). In some cases, one might observe a positive count and a zero coverage weight at the same time. Such counts arise through 'indirect' channels discussed above, and should be considered as significant underestimates. Among other things, this allows selecting a data sample based on a minimum threshold value of coverage. For example, consider only those counts derived from information that is $95 \%$ complete. However, applying such a 'filter' might involve a trade-off between geographical scope and data quality. 


\subsection{Protection in national jurisdictions through regional (international) patent filings}

Regional IP offices play a more-or-less important role in the world. For example, patenting at the European Patent Office (EPO) is an increasingly important route of protecting IP rights in Europe. Similarly, patenting at the two African regional IP offices (ARIPO, OAPI) also plays an important role. ${ }^{10}$ However, based on a regional application it is difficult to infer the true protection strategy of a patentee. As a consequence, when constructing patent indicators researchers frequently either treat EPO and other regional filings as if they were equivalent to national applications or simply set them aside as if they never existed. ${ }^{11}$

To address the potential biases, in this paper we propose a method to account for filings at the EPO by estimating the "protection propensities". We then use the propensities to apportion EPO counts onto national jurisdictions. And while here we focus on the EPO, this approach could potentially be extended to other regional offices subject to data availability.

\section{Apportionment of EPO patent filings using protection propensities}

There are several potential sources of data that are potentially useful for inferring patentee's market protection strategies, including (a) list of designated states using the PAT_EP database - a straightforward but not satisfactory solution because designation rules have changed over time and certain countries are designated 'by default' reducing the value of such data; (b) publication kind codes in PATSTAT that identify grants of patents - while recent additions to PATSTAT have made this approach more convenient ${ }^{12}$, it is not clear whether a validation of an EP patent in a member state is always associated with a corresponding record in PATSTAT; and (c) payment of validation and maintenance fees - the major conceptual upside is that the data should be a reliable indicator of patentees' preferences because they are associated with financial cost. The latter approach is discussed next.

In this paper, we use the European Patent Office's Inpadoc Legal Status database, also called Patent Register Service (PRS) Legal Status database, to construct "protection propensities" over time. The database contains data from 41 patent granting authorities going back to 1970. It contains over 120 million records, each referring to a distinct "legal status event", identified by a PRS code. The great advantage is that the PRS records are linked to PATSTAT.

There are several PRS codes that are potentially useful as evidence that patent applicants have designated, validated and maintained their patent rights in certain EPO member states against payment of fees.

In this paper we use data on post-grant fee payment (PGFP) to calculate the protection propensity (other alternatives that were also considered are summarized in the Annex). The major advantage of PGFP is that the data should be complete because patent offices have an interest in maintaining reliable records of fee payments; however, we identify several years and offices for which data in the PRS database are missing or appear incomplete. ${ }^{13}$ Another potential downside is the time lag due to granting of a patent and fee payment which causes problems for generating timely statistics. Therefore, we cannot calculate the protection propensities for the specific subset of relevant patents; instead we do so for all patents (PATSTAT total). More formally, the protection propensity is calculated as the ratio of a frequency count of EP applications that enter the national phase in a given member state as evidenced by payment of post-grant fees, and the frequency count of all (distinct) EP applications with PGFP evidence from any state. The protection propensity thus shows how often a typical EP application truly sought protection in a given member state (the sum of the propensities is thus more than 100\%). We construct such propensities for all member states for the 
time period starting with the year when they joined the EPO (Table 5). Propensities for selected offices are also shown in Figure 5.

The propensities implied by this statistic seem to be of reasonable magnitudes. Overall, four broad groups of member states can be distinguished: (i) the most frequently protected countries with propensities close to 50\%, including Germany, the United Kingdom and France, and followed by Italy (about 30\%); (ii) mid-size markets with propensities between $10 \%$ and $20 \%$, including Netherlands, Switzerland, Belgium, Sweden, Austria, and Spain; (iii) smaller markets with propensities between $1 \%$ and $10 \%$, including markets with stable propensities over time (e.g. Greece, Ireland, Portugal) as well as more dynamic markets whose propensities are growing quickly (e.g. Turkey, Finland, Poland); and finally (iv) markets with propensities of less than $1 \%$.

Note that over time there has been a progressive move from single country designation to automatic designation of eventually all EPO members in EPO applications after 2004. The legalistic interpretation would thus lead to apportioning all EPO member countries to every EPO application after 2004. However, such automatic (or default) list of designated countries inflates the "true" intentions of the applicants. Instead, in this paper we adopt the economic interpretation and seek to approximate what markets applicants really care about. Our objective is to apportion an EPO filing only to those member states where the applicant truly seeks to protect the invention. Evidence of postgrant fee payment is thus a suitable option. 
The Use of Patent Statistics for International Comparisons and Analysis of Narrow Technological Fields

Table 5. Protection propensities in EPO member states (1990-2012, 3-year moving average)

\begin{tabular}{|l|cccccccccccc}
\hline & 1990 & 1991 & 1992 & 1993 & 1994 & 1995 & 1996 & 1997 & 1998 & 1999 & 2000 & 2001 \\
\hline DE & 0.511 & 0.527 & 0.524 & 0.530 & 0.530 & 0.524 & 0.524 & 0.526 & 0.546 & 0.542 & 0.553 & 0.549 \\
GB & 0.522 & 0.532 & 0.525 & 0.522 & 0.508 & 0.504 & 0.505 & 0.511 & 0.506 & 0.513 & 0.524 & 0.538 \\
FR & 0.524 & 0.528 & 0.520 & 0.507 & 0.504 & 0.501 & 0.497 & 0.495 & 0.489 & 0.490 & 0.497 & 0.510 \\
NL & 0.363 & 0.344 & 0.337 & 0.337 & 0.323 & 0.301 & 0.283 & 0.271 & 0.268 & 0.268 & 0.269 & 0.273 \\
CH & 0.305 & 0.303 & 0.298 & 0.292 & 0.284 & 0.276 & 0.262 & 0.251 & 0.236 & 0.229 & 0.217 & 0.213 \\
BE & 0.300 & 0.295 & 0.289 & 0.285 & 0.267 & 0.252 & 0.235 & 0.228 & 0.221 & 0.220 & 0.212 & 0.209 \\
SE & 0.299 & 0.293 & 0.287 & 0.278 & 0.260 & 0.242 & 0.233 & 0.228 & 0.216 & 0.209 & 0.201 & 0.203 \\
AT & 0.218 & 0.217 & 0.214 & 0.214 & 0.204 & 0.194 & 0.185 & 0.181 & 0.174 & 0.173 & 0.171 & 0.170 \\
ES & 0.016 & 0.035 & 0.063 & 0.091 & 0.116 & 0.136 & 0.150 & 0.159 & 0.160 & 0.166 & 0.170 & 0.176 \\
LU & 0.135 & 0.136 & 0.135 & 0.135 & 0.125 & 0.116 & 0.103 & 0.098 & 0.091 & 0.089 & 0.083 & 0.082 \\
DK & & & & 0.008 & 0.018 & 0.030 & 0.042 & 0.051 & 0.058 & 0.064 & 0.066 & 0.071 \\
GR & 0.008 & 0.017 & 0.028 & 0.040 & 0.047 & 0.049 & 0.050 & 0.050 & 0.049 & 0.049 & 0.048 & 0.052 \\
IE & & & & & & 0.002 & 0.005 & 0.010 & 0.016 & 0.023 & 0.028 & 0.035 \\
PT & & & & & 0.001 & 0.003 & 0.007 & 0.013 & 0.018 & 0.024 & 0.029 & 0.034 \\
FI & & & & & & & & & & 0.001 & 0.003 & 0.006 \\
MC & & & & 0.000 & 0.000 & 0.001 & 0.004 & 0.006 & 0.010 & 0.012 & 0.013 & 0.015 \\
\hline Total & 3.200 & 3.228 & 3.221 & 3.238 & 3.186 & 3.130 & 3.086 & 3.080 & 3.058 & 3.070 & 3.082 & 3.134 \\
\hline
\end{tabular}

Table 5. (cont.)

\begin{tabular}{|c|c|c|c|c|c|c|c|c|c|c|c|c|}
\hline & 2002 & 2003 & 2004 & 2005 & 2006 & 2007 & 2008 & 2009 & 2010 & 2011 & 2012 & $\begin{array}{r}1990-2012 \\
\text { average } \\
\end{array}$ \\
\hline DE & 0.560 & 0.557 & 0.526 & 0.528 & 0.449 & 0.422 & 0.405 & 0.448 & 0.453 & 0.487 & 0.573 & 0.513 \\
\hline GB & 0.533 & 0.526 & 0.508 & 0.575 & 0.466 & 0.567 & 0.479 & 0.551 & 0.412 & 0.418 & 0.457 & 0.509 \\
\hline FR & 0.511 & 0.505 & 0.510 & 0.515 & 0.446 & 0.404 & 0.372 & 0.432 & 0.402 & 0.433 & 0.468 & 0.481 \\
\hline IT & & & & 0.185 & 0.265 & 0.342 & 0.250 & 0.287 & 0.289 & 0.346 & 0.285 & 0.281 \\
\hline NL & 0.267 & 0.253 & 0.227 & 0.212 & 0.155 & 0.165 & 0.149 & 0.175 & 0.139 & 0.119 & 0.148 & 0.245 \\
\hline $\mathrm{CH}$ & 0.206 & 0.199 & 0.181 & 0.170 & 0.132 & 0.119 & 0.112 & 0.131 & 0.179 & 0.157 & 0.162 & 0.214 \\
\hline BE & 0.201 & 0.197 & 0.181 & 0.170 & 0.120 & 0.108 & 0.102 & 0.130 & 0.122 & 0.111 & 0.105 & 0.198 \\
\hline SE & 0.197 & 0.191 & 0.188 & 0.154 & 0.109 & 0.096 & 0.121 & 0.145 & 0.123 & 0.105 & 0.104 & 0.195 \\
\hline AT & 0.164 & 0.159 & 0.148 & 0.138 & 0.105 & 0.095 & 0.090 & 0.106 & 0.104 & 0.098 & 0.129 & 0.159 \\
\hline ES & 0.180 & 0.184 & 0.185 & 0.178 & 0.148 & 0.142 & 0.151 & 0.179 & 0.170 & 0.165 & 0.181 & 0.144 \\
\hline LU & 0.078 & 0.080 & 0.074 & 0.066 & 0.046 & 0.040 & 0.038 & 0.045 & 0.049 & 0.044 & 0.038 & 0.084 \\
\hline DK & 0.073 & 0.077 & 0.076 & 0.066 & 0.048 & 0.045 & 0.054 & 0.061 & 0.088 & 0.085 & 0.082 & 0.058 \\
\hline GR & 0.053 & 0.056 & 0.055 & 0.056 & 0.046 & 0.039 & 0.043 & 0.053 & 0.057 & 0.044 & 0.037 & 0.045 \\
\hline $\mathrm{IE}$ & 0.041 & 0.048 & 0.052 & 0.051 & 0.042 & 0.037 & 0.045 & 0.056 & 0.058 & 0.056 & 0.057 & 0.037 \\
\hline PT & 0.039 & 0.043 & 0.046 & 0.042 & 0.031 & 0.029 & 0.035 & 0.043 & 0.044 & 0.030 & 0.046 & 0.029 \\
\hline $\mathrm{FI}$ & 0.013 & 0.020 & 0.026 & 0.029 & 0.025 & 0.026 & 0.030 & 0.039 & 0.038 & 0.042 & 0.046 & 0.025 \\
\hline TR & & & 0.002 & 0.005 & 0.006 & 0.008 & 0.012 & 0.018 & 0.027 & 0.036 & 0.035 & 0.016 \\
\hline$M C$ & 0.018 & 0.022 & 0.024 & 0.024 & 0.018 & 0.016 & 0.017 & 0.020 & 0.020 & 0.019 & 0.018 & 0.014 \\
\hline $\mathrm{CZ}$ & & & & 0.001 & 0.001 & 0.004 & 0.007 & 0.012 & 0.024 & 0.024 & 0.030 & 0.013 \\
\hline PL & & & & & & 0.001 & 0.004 & 0.007 & 0.012 & 0.018 & 0.027 & 0.012 \\
\hline $\mathrm{CY}$ & 0.001 & 0.004 & 0.006 & 0.008 & 0.006 & 0.007 & 0.009 & 0.013 & 0.015 & 0.013 & 0.012 & 0.008 \\
\hline RO & & & & & & 0.001 & 0.003 & 0.005 & 0.010 & 0.013 & 0.013 & 0.008 \\
\hline $\mathrm{HU}$ & & & & 0.000 & 0.001 & 0.002 & 0.004 & 0.007 & 0.010 & 0.013 & 0.015 & 0.006 \\
\hline SK & & & & 0.000 & 0.001 & 0.002 & 0.004 & 0.006 & 0.009 & 0.011 & 0.013 & 0.006 \\
\hline
\end{tabular}


The Use of Patent Statistics for International Comparisons and Analysis of Narrow Technological Fields

\begin{tabular}{|c|c|c|c|c|c|c|c|c|c|c|c|c|}
\hline SI & & & & 0.000 & 0.000 & 0.001 & 0.003 & 0.005 & 0.006 & 0.008 & 0.010 & 0.004 \\
\hline BG & & & & 0.000 & 0.000 & 0.001 & 0.002 & 0.005 & 0.006 & 0.008 & 0.009 & 0.004 \\
\hline $\mathrm{EE}$ & & & & 0.000 & 0.000 & 0.001 & 0.003 & 0.004 & 0.005 & 0.006 & 0.007 & 0.003 \\
\hline IS & & & & & & & 0.000 & 0.001 & 0.002 & 0.003 & 0.004 & 0.002 \\
\hline NO & & & & & & & & & & 0.001 & 0.002 & 0.002 \\
\hline LV & & & & & & & & 0.001 & 0.001 & 0.002 & 0.003 & 0.002 \\
\hline LT & & & & & & 0.000 & 0.000 & 0.001 & 0.002 & 0.003 & 0.004 & 0.002 \\
\hline MT & & & & & & & & & & & 0.001 & 0.001 \\
\hline HR & & & & & & & & & & 0.001 & 0.001 & 0.001 \\
\hline$A L$ & & & & & & & & & & & & \\
\hline LI & & & & & & & & & & & & \\
\hline MK & & & & & & & & & & & & \\
\hline RS & & & & & & & & & & & & \\
\hline SM & & & & & & & & & & & & \\
\hline Total & 3.132 & 3.121 & 3.013 & 3.174 & 2.664 & 2.722 & 2.543 & 2.988 & 2.876 & 2.916 & 3.118 & 3.317 \\
\hline
\end{tabular}

Figure 5. Propensity to protect in an EPO member state (PGFP, 3-year MA)

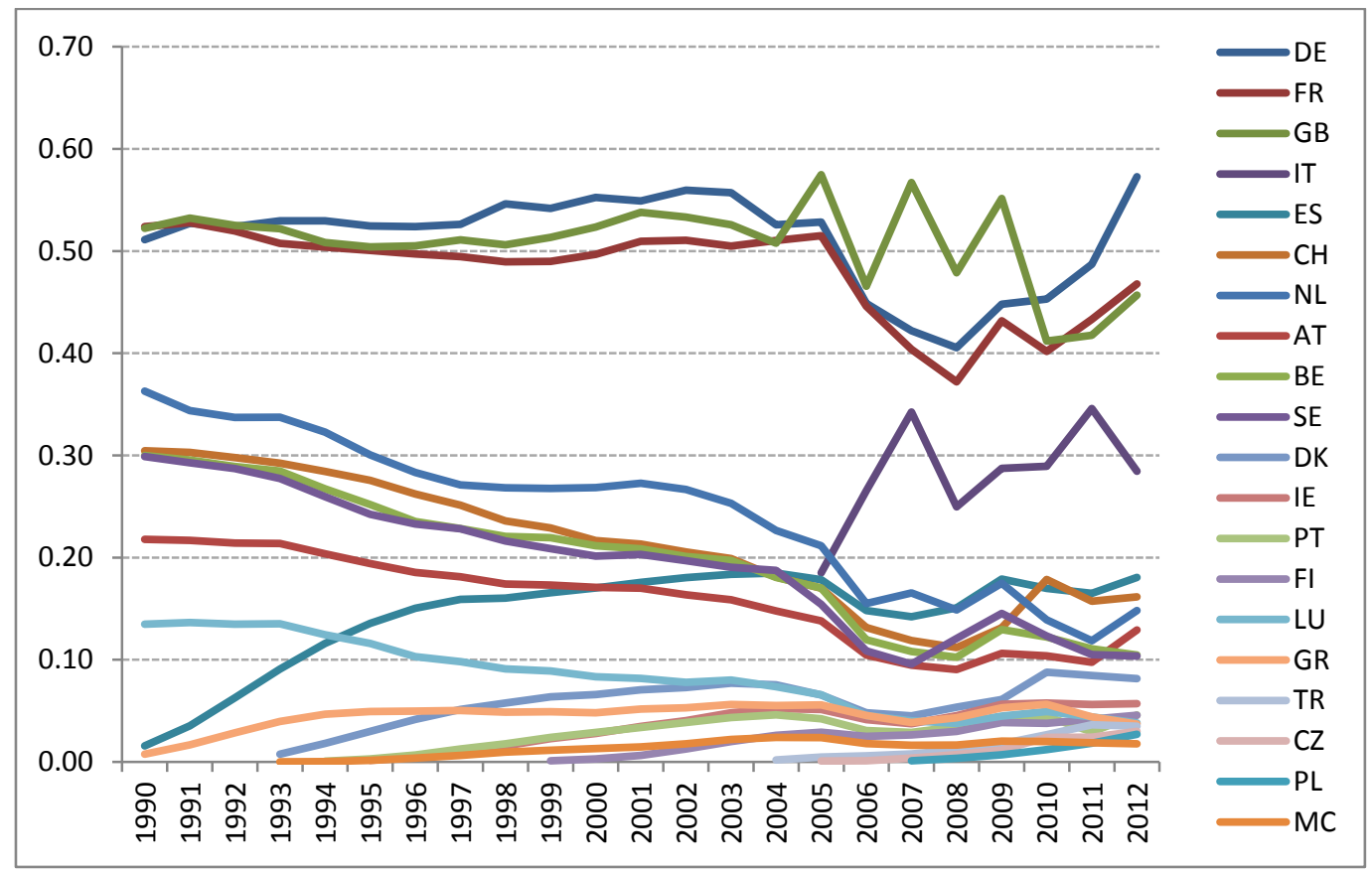

In this paper, the protection propensities are generated only over time, assuming that all patent applicants in a given year are homogeneous with respect to protection propensities. This could be refined further by allowing the propensities to vary not only over time but also across priority office as well as duplicate office, inventor country or applicant country (although such information is sometimes missing, reducing the sample size). EPO applications could then be apportioned along all these vectors.

The PRS data necessarily involve certain time lags. Therefore, in this paper we construct summary statistics of protection propensities for TOTPAT and apply these on EPAT. This is based on the assumption that protection propensities do not vary systematically across technological fields. We 
thus use the full sample of PRS data (all patents, not only environmental) to calculate the propensities. We then apply the propensities on the "environmental" counts and apportion EPO applications onto national patent offices of the member countries (as unit counts, not fractionally). Figure 6 shows the evolution over time of national and EP-apportioned patenting activity in air pollution abatement technologies for selected countries. An alternative approach would be to estimate the protection propensities econometrically as a function of characteristics of patent applicants, inventors, priority office, and technological field, and then nowcast the recent years.

Figure 6. Patenting activity at the national office versus EP-apportioned filings
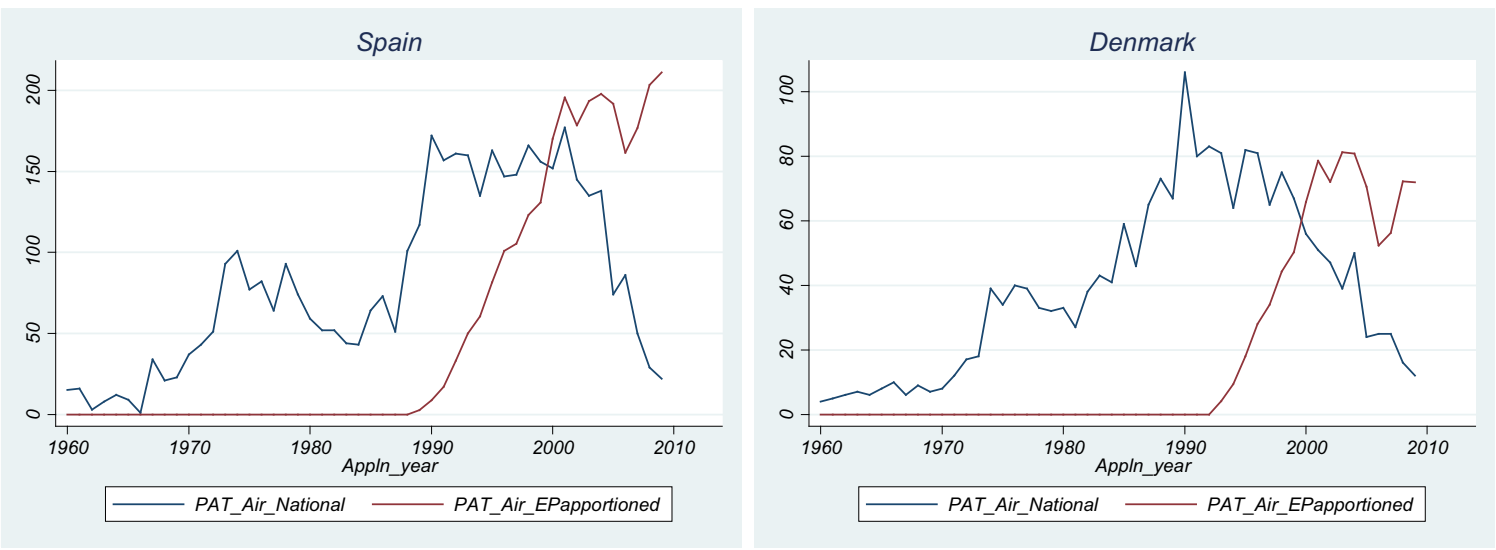

In sum, construction of 'protection propensities' allows apportioning of regional patent filings onto national jurisdictions, and thus allows to correct for the drop in national patenting caused by growing tendency to protect inventions at the regional rather than national authorities. Subject to availability of protection data, this methodology could be extended to other regional offices.

Such EPO protection propensities can be useful for at least two types of applications. First, the propensities can be used to estimate patent family size when constructing patent family-based indicators. Consider the following example using data from Table 5: Summing across protection propensities of all EPO member states in a given year yields the "aggregate propensity" - that is, the average size of an EP patent family. For most of the 1990-2012 period the aggregate propensity varies between 2.5 and 3.2 (last row in Table 5). For example, in year 2000 this is estimated to be 3.082, so a singleton application deposited in that year at the EPO corresponds, on average, to a patent family with an approximate family size of 3. However, if PATSTAT also contains a trace of a German family member then the German application must contribute its full weight (unity) rather than its estimated weight in that year (1 rather than 0.553). Therefore, the equivalent size of such patent family is approximately 3.5 (Table 6 summarises this example).

Table 6. Estimation of patent family size using EP protection propensities

\begin{tabular}{lr}
\hline Observed family & Estimated family size in year 2000 \\
\hline$E P$ singleton & 3.082 \\
$E P+D E$ & $3.082-0.553+1=3.530$ \\
$E P+$ US & $3.082+1=4.082$ \\
\hline
\end{tabular}

Second, the protection propensities can be used to apportion patenting activity onto national jurisdictions of EPO member states, for example to construct patent stocks to be used in empirical analyses (Figure 7). 
Figure 7. Technology stock based on national and EP-apportioned patents (15\% discount rate)
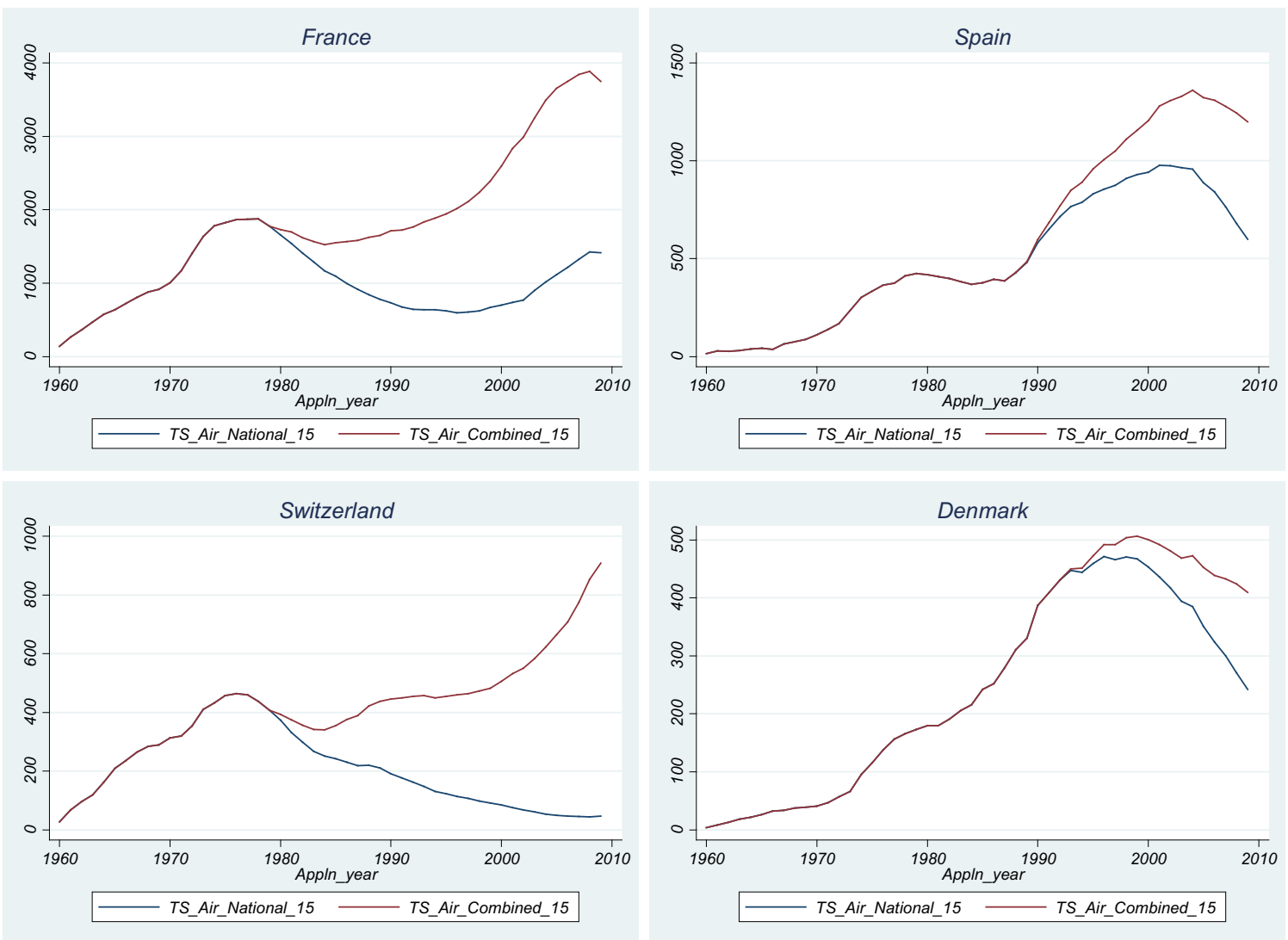

\subsection{Missing information}

For narrow technological fields, small inventor countries, or countries with low levels of patenting activity - that is, situations with frequent zeros and generally low patent counts - increasing the 'yield' of patent data extractions is valuable. However, this should be done in a manner that minimizes a potential bias in the analysis (this will be discussed in Section 4 below).

\section{Imputing missing information on inventors}

One way to increase the magnitude and variation of counts is to impute missing inventor information. While basic bibliographic information such as application authority and application date is always available in patent databases, additional descriptive information such as name and address of the inventor(s) and applicant(s) are often missing or incomplete. To mitigate this problem we retrieve inventor data for all patent family members and impute from duplicate applications information on inventor countries that is not listed in the priority document. The imputed inventor information is then stored at the level of the priority because it is common to all members of the single-priority patent family. This typically allows increasing the volume of data with known inventor information by 3 to 4 percentage points (Table 7). ${ }^{14}$

While technically the same imputation procedure could be conducted to mitigate missing applicant information, conceptually it might be preferable to retain all sets of applicant data within a family because applicants might be different for each patent application, i.e. due to change of ownership. 
The Use of Patent Statistics for International Comparisons and Analysis of Narrow Technological Fields

Table 7. Benefit of imputing inventor information from duplicate filings

\begin{tabular}{|l|l|l|}
\hline & $\begin{array}{l}\text { Priorities with known } \\
\text { inventor country }\end{array}$ & $\begin{array}{l}\text { Priorities with known } \\
\text { inventor country } \\
\text { retrieved within PATSTAT }\end{array}$ \\
\hline Renewable energy $(Y 02 E 10)^{1}$ & $42.3 \%$ & $46.2 \%(+3.9)$ \\
\hline Geothermal energy $(Y 02 E 10: 1)^{1}$ & $54.0 \%$ & $58.1 \%(+4.1)$ \\
\hline Wind power (Y02E10:7) ${ }^{1}$ & $52.6 \%$ & $55.7 \%(+3.1)$ \\
\hline Wind power (F03D) ${ }^{2}$ & $53.2 \%$ & $56.3 \%(+3.1)$ \\
\hline (Waste)water treatment (CO2F) $^{2}$ & $35.3 \%$ & $38.8 \%(+3.5)$ \\
\hline Notes: \\
$\begin{array}{l}{ }^{1} \text { Based on searches in APPLN_ECLA table } \\
{ }^{2} \text { Based on searches in both APPLN_ECLA and APPLN_IPC table }\end{array}$
\end{tabular}

Imputing missing information on patent classifications

Another source of missing information are missing classification symbols. For search strategies defined in terms of IPC symbols, the yield can be increased by searching also in the APPLN_ECLA (more recently APPLN_CPC) table in addition to APPLN_IPC table. This is because EPO examiners often assign ECLA symbols even to foreign filings if they consider that the existing classifications are not sufficient or appropriate. For example, in the case of wastewater treatment technologies this allows increasing the volume of data by 3 percentage points compared with a search conducted only in the APPLN_IPC table (Table 8).

Table 8. Benefit of imputing IPC symbols using the APPLN_ECLA table

\begin{tabular}{|l|c|c|c|}
\hline & Nb. of documents identified (appln_id's) \\
\hline & $\begin{array}{c}\text { search in } \\
\text { APPLN_IPC only }\end{array}$ & $\begin{array}{c}\text { search in both } \\
\text { APPLN_IPC \& } \\
\text { APPLN_ECLA }\end{array}$ & $\begin{array}{c}\text { search in } \\
\text { APPLN_ECLA only }\end{array}$ \\
\hline (Waste)water treatment (CO2F) & 433,698 & 448,427 (+3\%) & 199,435 \\
\hline Wind energy (Y02E10/7) & - & - & 62,702 \\
\hline Wind motors (F03D) & 64,339 & $69,476(+8 \%)$ & 43,151 \\
\hline Climate mitigation in transport (Y02T) & - & - & 293,670 \\
\hline Electric \& hybrid cars (IPC-based) & 113,038 & $128,991(+14 \%)$ & 71,357 \\
\hline Climate mitigation in buildings (Y02B) & - & - & 209,898 \\
\hline Energy efficiency in buildings (IPC-based) & 260,855 & $289,178(+11 \%)$ & 134,912 \\
\hline
\end{tabular}

\section{Expansion of patent families}

To construct patent families, we proceed in three steps. First, the patent database is searched for the relevant (e.g. "green" or "environment-related") patents using a given definition - a search strategy. Second, we search for potential family members outside of this subset of documents using the DOCDBFAM table that gives patent applications protecting the same set of claims. Third, we identify priority relationships within this enlarged subset of patent documents. 
Figure 8. Expansion of patent families

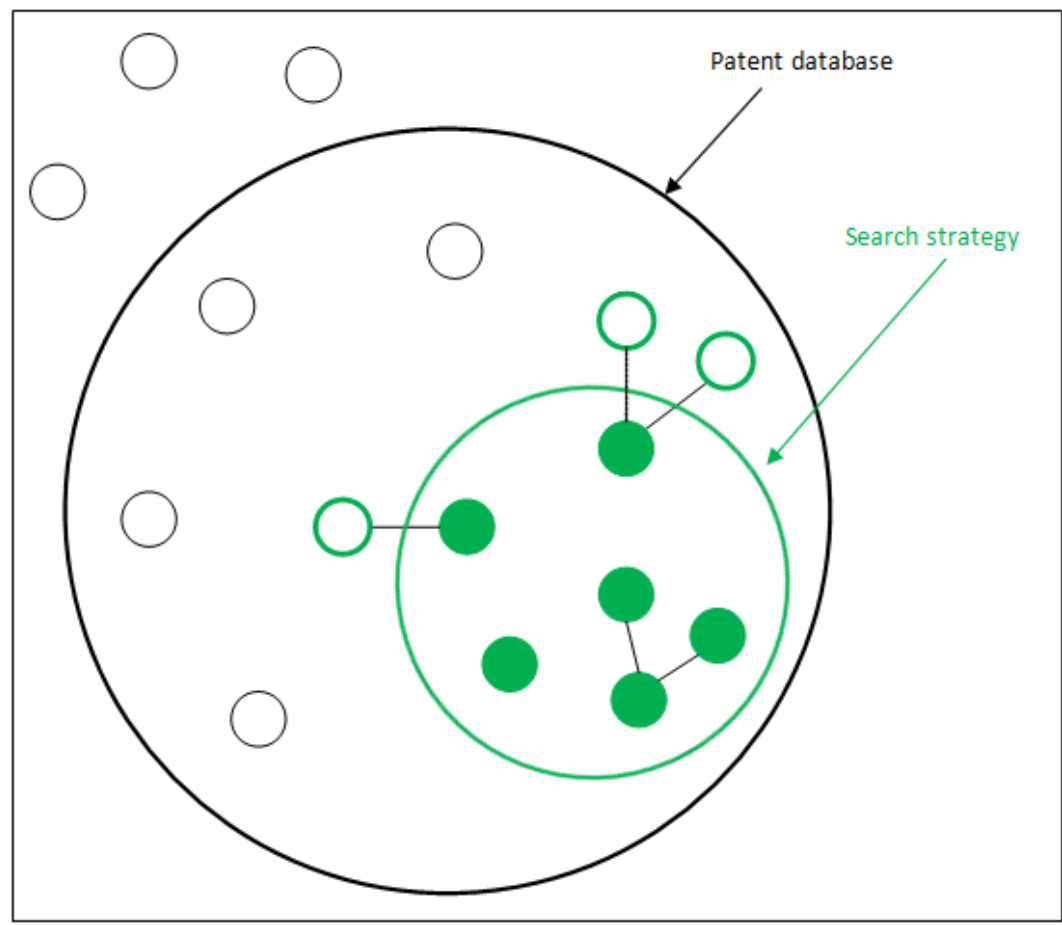

Table 9. Benefit of expansion: Additional patent family members identified

\begin{tabular}{|l|c|c|}
\hline & Within & Outside \\
\cline { 2 - 3 } & Appln_id's & $\begin{array}{c}\text { Full expansion } \\
\text { (additional Appln_id's) }\end{array}$ \\
\hline Wind energy (Y02E10/7) & 62,702 & \\
Wind motors (FO3D) & 69,476 & $3,879(+5 \%)$ \\
\hline
\end{tabular}

This approach also helps to mitigate the bias historically created by the distinction between coreand advanced-level IPC classes and the differentiated obligations of patent offices to classify in the latter (only patent offices in the "PCT-minimum requirements" are mandated to classify at the advanced level). Hence, when a search strategy is based on advanced-level IPC symbols (which is almost always the case), small patent offices that only classify at the core level would be underrepresented in the searches. The next Section discusses this issue in greater detail.

\section{Constructing variables to normalize (or control for) missing information}

While strategies to impute missing information are valuable because they increase the "yield" of patent extractions, they too might introduce potential biases in analysis. So both, the presence of missing information, as well as imputation thereof, might give rise to biases. One way to mitigate them is to generate a corresponding count of patent "totals" (so-called TOTPAT). By corresponding we mean a statistic that is constructed in a manner that is identical to the "environmental" count (EPAT) with the one exception that all documents (not only "environmental") are considered. For example, if missing information on inventors is imputed, this should be done identically for EPAT as for TOTPAT. Ways in which such TOTPAT variable can be used for the purposes of normalization in descriptive analyses or as a control variable in econometric analyses will be discussed in Section 4 below. 
Assignment of patent classification symbols to patented inventions is not homogeneous across patent offices and this is another potential source of idiosyncratic biases in patent analysis. Questions arise concerning the best ways to apply a given search strategy to identify the relevant patents in a patent database; and, how to identify the relevant population, or a relevant subset in an unbiased manner? ${ }^{15}$

The use of the international patent classification system (IPC) varies across offices to a certain extent. For example, while patent offices listed in the "PCT minimum requirements" are obliged to assign IPC symbols at the 'advanced' level, other patent offices must do so only at the 'core' level although in practice they might assign advanced level symbols in areas of particular interest. This is important because search strategies for narrow technological fields are often based on symbols at the advanced level of IPC. Such heterogeneous practices in assignment of IPC symbols thus create a potential source of a bias.

Even more importantly, many national patent offices have developed their own patent classification systems (ECLA, USPC, F-terms, etc.) to classify patent applications, the related prior art, and perhaps patents in selected domains that are of specific interest to their respective countries. In any case, the national patent classification symbols are assigned only to a certain subset of the world's "population" of patent applications. For example, the EPO uses the ECLA symbols ${ }^{16}$ to systematically classify all patent documents from the "PCT minimum requirements" in one of the EPO official languages (incl. German, English and French), with documents filed in other languages (e.g. Japanese, Russian, Spanish) are excluded. Patent documents in Dutch (e.g. those filed in the Netherlands, Belgium and Luxembourg) are also classified as well as those with first filings residents from Austria, Australia and Canada. In addition, Korean, Chinese and Indian documents are classified if they are in one of the four EPO languages. Additional documents might be machine-translated if they are in selected priority areas (determined based on IPC symbols assigned by home offices). Finally, some Japanese documents were classified in the past based on abstracts, and they might be re-classified if a subsequent equivalent is published in one of the four EPO languages. ${ }^{17}$ The situation is in many respects similar, if not "worse", at other patent offices worldwide because they too face resource constraints.

In sum, while the practice of selective classification in national systems might be economically justified, from a researcher's point of view it creates another potential source of bias. For example, while a large majority of patent documents in PATSTAT have an ECLA symbol assigned, it is not true of all the patent documents. Therefore, a search strategy that uses an ECLA symbol might yield biased results insofar it cannot identify relevant documents among those without an ECLA symbol.

The situation is even more complicated with search strategies that use the recently introduced Y02 class (so-called Y-tags) developed by the EPO. While this new tagging scheme greatly facilitates identification of many climate-related inventions by non-specialists, users should be aware of its implications. ${ }^{18}$ To be clear, the new tagging scheme does not substitute for the standard ECLA (or CPC) classification symbols, rather it is complementary. Technically, the tagging does not involve examination of each individual patent application, rather it is constructed as a set of search algorithms that exploit the full stock of descriptive attributes in DOCDB ${ }^{19}$, including the IPC and ECLA symbols, as well as EPO's internal schemes of in-computer-only (ICO) classification symbols and keywords (KW). In addition, for some technologies keyword searches on English titles and abstracts are conducted (either as a single keyword or in combination). Finally, where available, Derwent WPI index might be used as well. ${ }^{20}$ Therefore, the population of patents from which such searches draw is 
unknown (or imperfectly defined, at least). Next we discuss strategies that help mitigate any potential biases.

\section{Constructing variables to normalize (or control for) non-systematic classification}

Ideally, every "environmental" patent (EPAT) search strategy needs its own corresponding TOTPAT control variable. A researcher using an IPC-based (or ECLA-based) search strategy can easily generate a corresponding count of patent "totals" (e.g. all patents with an/any IPC (ECLA) symbol assigned). Similarly, if a search strategy is based on English keywords in titles and abstracts, then the corresponding TOTPAT would be constructed as a count of all patent families with an English-language title or abstract. In these cases, construction of such variables is straightforward because the set of patents from which such searches draw is known - PATSTAT allows identifying these relevant sets of documents (see Table 10). At the aggregate level TOTPAT_IPC and TOTPAT_ECLA are highly correlated because as many as $80 \%$ of all documents (appln_id's) in PATSTAT have both IPC and ECLA symbols assigned. However, there are important differences at the more disaggregated level.

However, in the case of a Y-based search strategy the set of "potentially identifiable" documents is only imperfectly defined, and therefore it is more difficult to generate a corresponding "total". As described above, the set of documents that could have theoretically been searched and assigned a given Y-tag is only imperfectly defined. Therefore, constructing TOTPAT_YTAG correctly is an impossible task, unless a separate TOTPAT count is generated for every Y-symbol which is in most practical uses of patent data too costly. ${ }^{21}$ In most practical applications, the researcher might use an approximation of TOTPAT_YTAG (\#4) based on some combination of the corresponding totals - for example, the union of the IPC and ECLA TOTPATs (\#5 in Table 10). 
Table 10. Construction of corresponding totals

\begin{tabular}{|c|c|c|c|}
\hline & $\begin{array}{l}\text { If EPAT search } \\
\text { strategy is based } \\
\text { on: }\end{array}$ & $\begin{array}{l}\text {...then TOTPAT should be } \\
\text { constructed as: }\end{array}$ & $\begin{array}{l}\text { Share of appln_id's } \\
\text { in PATSTAT }\end{array}$ \\
\hline (1) & IPC symbols & $\begin{array}{l}\text { All documents (single-priority } \\
\text { patent families) that are } \\
\text { 'identifiable' using IPC symbols } \\
\text { (i.e. all appln_id's listed in the } \\
\text { APPLN_IPC table). }\end{array}$ & $79 \%$ \\
\hline (2) & ECLA symbols & $\begin{array}{l}\text { All documents (single priority } \\
\text { patent families) that are } \\
\text { 'identifiable' using ECLA symbols } \\
\text { (i.e. all appln_id's listed in the } \\
\text { APPLN_ECLA table with EC as } \\
\text { classification scheme). }\end{array}$ & $49 \%$ \\
\hline (3) & $\begin{array}{l}\text { Keyword searches } \\
\text { on titles and/or } \\
\text { abstracts }\end{array}$ & $\begin{array}{l}\text { All documents (single priority } \\
\text { patent families) that are } \\
\text { 'identifiable' using keyword } \\
\text { searches (i.e. all documents with } \\
\text { title/abstract in the corresponding } \\
\text { language). }\end{array}$ & $\begin{array}{c}58 \% \\
\text { (English) }\end{array}$ \\
\hline (4) & Y02 tags & $\begin{array}{l}\text { All documents (single-priority } \\
\text { patent families) that could have } \\
\text { potentially been tagged by the } \\
\text { EPO. }\end{array}$ & $\begin{array}{c}? \\
\text { (this will vary by } \\
\text { individual Y-symbol) }\end{array}$ \\
\hline (5) & $\begin{array}{l}\text { IPC and ECLA } \\
\text { symbols }\end{array}$ & The union of (1) and (2) above. & $80 \%$ \\
\hline (6) & $\begin{array}{l}\text { IPC, ECLA, ICO, or } \\
\text { English title/abstract }\end{array}$ & $\begin{array}{l}\text { The union of the respective } \\
\text { counts. }\end{array}$ & $84 \%$ \\
\hline
\end{tabular}

For these reasons, and when there is a choice between an IPC-based and Y02-based search strategy, it might be preferable to use IPC because it allows correctly specifying the "counterfactual" (all patents with and assigned IPC symbol, not only the 'environmental' IPC symbol), a task that is more difficult (if not impossible) to do for Y-tagging because the individuals to be tagged are not drawn randomly from this population (we do not know the 'taggable' population). This might be particularly important for regions of the world that are not well covered by the Y02 scheme.

This problem will diminish over time because many of the elements (ECLA, ICO, KW) have now become part of the new CPC system. As patent classification systems become increasingly harmonised, and the CPC is used more widely, the precision of TOTPAT_YTAG will approach that of TOTPAT_CPC. Until then, for research hypotheses where it is of key importance to have a precise control (counterfactual) IPC might indeed be the better option.

In some cases it might be possible to define a "sectoral total" instead of a "PATSTAT total" as discussed above. For example, analysis of electric and hybrid cars patenting can be complemented with a "total" count for the entire sector of motor vehicles or road vehicles which it is possible to define using IPC symbols (Haščič and Johnstone 2011; see also Aghion et al. 2012). Similarly, analysis of fuel-efficient energy generation technologies can be complemented with a "total" count for the entire sector of energy generation from fossil fuel combustion (Lanzi et al. 2011; Noailly and Smeets 2013). 


\section{IMPLICATIONS FOR EMPIRICAL ANALYSIS}

The need for a normalization count (control variable)

Dealing with a narrow technological field is both a boon and a bane. On the one hand, this poses certain difficulties in terms of indicator construction (Section 2), but on the other hand it allows generating a "total" variable that is an appropriate answer to these, and other, difficulties. The conceptual justification for the use of such variable is twofold: First, there are conceptual (economic) reasons for using a TOTPAT arising out of the need to control for many generic factors that affect patenting in a narrow field, including:

- Differences in inventive capacity

- Differences in propensity to patent

- Differences in patent breadth and patent 'quality'

- Other factors that might affect patenting in general

Obviously, there might be suitable substitutes for TOTPAT to control for these differences across countries, sectors, firms, and over time (while such substitutes may be even better, the data is not always readily available). ${ }^{22}$

Second, there is another set of reasons that might be even more important and for which it is impossible to substitute with other data. These are the methodological (idiosyncratic) reasons calling for the use of a TOTPAT arising out of:

- Incomplete information due to differences in coverage of patent databases (Section 3.1)

- Imperfect information on jurisdictions where patent protection is sought through regional procedures (Section 3.2)

- Extent of missing information on inventors, applicants, patent classes (Section 3.3)

- Non-systematic classification of patent documents (Section 3.4)

Using a TOTPAT variable deals perfectly with the latter (idiosyncratic) sources of biases (except for those discussed in 3.4) and imperfectly with the former (conceptual) sources of biases. How exactly should such a TOTPAT variable be constructed will depend on three factors:

i) Indicator selected (e.g. PF2 for invention, PF1 for co-invention)

ii) Search strategy used (e.g. based on IPC, ECLA, keyword searches, or other)

iii) Algorithm used to construct the EPAT count (i.e. treatment of idiosyncrasies, other programming details that might affect the final outcome).

The corresponding TOTPAT is identical to EPAT in all the three aspects above. It is measured using the same indicator (e.g. PF2, TPF, count of co-inventions), based on the same type of search strategy (see Table 10), and constructed using an otherwise identical algorithm.

In descriptive analyses, inclusion of a TOTPAT variable provides suitable context and a basis for interpretation of trends observed in EPAT. Another option is to 'normalize' the EPAT count, for example, as a measure of specialisation expressed as a ratio of a country's share of EPAT on TOTPAT 
over the share of EPAT over TOTPAT worldwide (relative propensity to invent, relative propensity to patent) although such measures might be difficult for a layperson to interpret.

However, this approach yields the greatest benefits in econometric analyses. Indeed, many of the potential biases discussed above can be successfully mitigated by inclusion of a TOTPAT control variable (of the same dimensionality as EPAT). Such variable has the potential to partially control for changes in patenting propensities over time and across countries and offices, and to correct measurement error due to the idiosyncrasies detailed above. In empirical research one might be tempted to disregard such potential errors; while they might indeed be negligible in the EU-US space, shifting the analysis outside of the OECD countries increases the potential for erroneous conclusions, and hence the need to control for potential idiosyncratic biases.

\section{CONCLUDING REMARKS}

This paper draws attention to several issues that are specific to analysis of narrow technological fields in cross-country international comparisons. Moreover, there is a rising interest in patent analyses in developing countries and this raises new types of issues. First, the paper advocates the use of a range of patent indicators based on international patent family size. While it concludes that determining the "optimal" family size for a given application is largely an empirical question, PF2 or higher-order restrictions are preferable than PF1, unless the technological field studied is so narrow that this restricts the variation in the data 'too much' (too many zeros and low counts). In such cases one has no other option but to use PF1. There is indeed a trade-off between patent quality and breadth of technological fields studied.

Second, the paper reviews four types of idiosyncratic problems in the underlying data, including how to tell zeros from missings; how to address the problem of regional patent filings; how to increase yield of database extractions; and how to face patent identification problems. The paper advocates using a normalization (or control) variable constructed in an analogous manner as the indicator of primary interest. All patent databases come with a warning - do not blindly estimate on the contents of the database! 


\section{NOTES}

1 PCT count is not always a suitable indicator for inter-country comparisons because the propensity to use the PCT route varies across countries and over time (even after year 2000). Inventors from different countries vary in their likelihood of pursuing the PCT through to the national/regional phase (e.g. 80\% for NL, 30\% RU and $\mathrm{KR}$ ), and as such PCT counts suffer from their own specific type of 'home bias'. It is equivalent to a call option rather than a true patent application, and because the initial costs are rather low (esp. in the international phase) PCT applications often covers inventions of little value (OECD 2009).

2 Working with patent families is essential to avoid double-counting of inventions when patent data from multiple patent offices are pooled together. We adopt the notion of single-priority patent family (Martinez 2010) because it facilitates many of the steps discussed herein. We calculate the "international patent family size" that is, the number of distinct patent authorities within a family. As such, we distinguish between 3 types of equivalents - singleton priorities, claimed priorities and duplicates.

3 Claimed priorities with regional weights would yield a “dyadic patent family” indicator.

4 In the same vein, de Rassenfosse et al. (2013) advocate a metric based on "a worldwide count of priority patents" as a measure of inventive activity (equivalent to PF1 presented here). They highlight the advantages of such a measure relative to PCT counts.

5 Claimed priorities account for a relatively small proportion of the stock of patent applications. For example, in a study focusing on innovation in climate change mitigation technologies found that only about $11 \%$ of the relevant stock of patent applications included in PATSTAT were CPs, with 34\% being their duplicates, and 55\% being singletons (Haščič et al. 2010). In other words, in climate change mitigation technologies CPs represent only $16 \%$ of the stock of patented inventions (simple patent families), while the large majority (84\%) of these inventions were only protected at a single patent office (singletons). It must be noted that there is variation in these proportions across patent offices.

6 Note that account has to be taken of changing membership through time.

7 For example, former Eastern Germany (DD) complete data until 1999; former Czechoslovakia (CS) complete data until 1993, thereafter complete data separately for SK and CZ; former Yugoslavia (YU) data until 1992.

8 The statistical data for Israel are supplied by and under the responsibility of the relevant Israeli authorities. The use of such data by the OECD is without prejudice to the status of the Golan Heights, East Jerusalem and Israeli settlements in the West Bank under the terms of international law.

9 a) Note by Turkey:

The information in this document with reference to "Cyprus" relates to the southern part of the Island. There is no single authority representing both Turkish and Greek Cypriot people on the Island. Turkey recognises the Turkish Republic of Northern Cyprus (TRNC). Until a lasting and equitable solution is found within the context of the United Nations, Turkey shall preserve its position concerning the "Cyprus issue".

b) Note by all the European Union Member States of the OECD and the European Union:

The Republic of Cyprus is recognised by all members of the United Nations with the exception of Turkey. The information in this document relates to the area under the effective control of the Government of the Republic of Cyprus.

10 ARIPO and OAPI together accounted for 34\% of patent applications deposited in Africa during 1980-2009, excluding the South African office (Haščič et al. 2012).

11 The ignorance of the "do nothing" approach is not necessarily bad as long as one is comfortable with its implications, such as (i) wrongly classifying an EP patent application as a singleton when in fact it is a claimed 
priority, or (ii) wrongly classifying a patent family as a claimed priority when in fact it is only a singleton (e.g. consider a patent family consisting of EP and DE applications with DE as the only designated country).

12 Previously, one needed to determine whether a patent has been granted at each of the 38 member states patent authorities, hence making sense of the 38 different publication kind code systems. Fortunately, this has been much facilitated in recent editions of PATSTAT.

13 Specifically, data for years prior to 1990 seem rather incomplete; therefore we only construct the propensities for the period after 1990 (for Italy only after 2004). Even after 1990, data for some years and offices are missing, with two different situations occurring: (1) A year count is missing (GR 2007, LT 2007) and years around seem to be correct (the same trend before and after the missing year). In this case we simply impute a value for the missing year corresponding to the mean of the year before and the year after. (2) A year count is missing (FR 2007, IT 2007) and the years before and after show implausibly high numbers above the long-term trend. In this case we assume that for an unknown reason the patents have been distributed among the years before and after the missing year. ). In this case we re-allocate a third of the count proceeding the missing year, and a third of count in the year following the missing year. Moreover, the total on which the propensities are calculated also has to be corrected (the total for 2007 is very low compared with other years). The total is the count of distinct applications among all countries (no double-counting for applications with protection in multiple states). We use the "duplicate protection" coefficient observed in 2005 and 2009, take the mean of those two values, and divide through the values re-allocated from 2006 to 2007 and from 2008 to 2007 to inflate the total for 2007.

${ }^{14}$ A similar approach was developed by De Rassenfosse et al. (2013).

15 Development of a suitable search strategy - that is, a mapping between patent classification systems and a definition of "environmental" innovation - is a distinct problem and is not addressed here. A companion paper is devoted to this issue.

16 Starting 2013 the EPO and USPTO have started using a common classification system (CPC) and consequently the use of ECLA and USPC is being discontinued.

17 Personal communication from Mr. Pierre Held of the European Patent Office, November 2012 in Hamburg, Germany.

18 The benefit of the new Y02 scheme is twofold. First, it allows identifying patent documents in technological fields that would otherwise not be possible by a non-specialist! The tags were developed by patent examiners at the EPO who are specialised in the different fields covered. Second, it provides a much greater level of detail than what would be possible using the IPC. This is a significant advantage over previous searches based on the IPC. See Veefkind et al. (2012) for further details.

19 DOCDB, also referred to as EPODOC, is the EPO's master documentation database. This is the resource used by patent examiners for searches of prior art.

20 Personal communication by Mr. Victor Veefkind of the European Patent Office, 2012.

21 In the ideal case, one would construct a different TOTPAT for each of the individual Y02 symbols depending on type of attributes used in the algorithm to assign a given symbol (e.g. some algorithms use keywords, others do not). While such information could be made available, it is of limited practical use because (i) analysis is rarely conducted at the level of an individual symbol (e.g. Y02E10:43 Fresnel lenses); rather, it is the hierarchically more aggregated level (e.g. Y02E10:4 Solar thermal energy) that is of interest to decisionmakers. In such case it is not clear how can one generate a TOTPAT at the aggregate level when individual tags use different sets of attributes; (ii) Even if the same set of attributes were used within a given "field" of technology, analyses often compare several of such "fields" implying the use of multiple "totals" (e.g. a total for solar energy, a total for wind energy, etc.).

22 See Johnstone et al. (2012) for a discussion. 


\section{REFERENCES}

Aghion P., Dechezleprêtre, A., Hemous, D., Martin, R. and J. Van Reenen (2012) "Carbon Taxes, Path Dependency and Directed Technical Change: Evidence from the Auto Industry," NBER Working Papers 18596, National Bureau of Economic Research.

Barton J.H. (2007), “Intellectual Property and Access to Clean Energy Technologies in Developing Countries”, International Centre for Trade and Sustainable Development (ICTSD).

Belward A., B. Bisselink, K. Bódis, A. Brink, J.-F. Dallemand, A. de Roo, T. Huld, F. Kayitakire, P. Mayaux, M. Moner-Girona, H. Ossenbrink, I. Pinedo, H. Sint, J. Thielen, S. Szabó, U. Tromboni, L. Willemen (2011), "Renewable energies in Africa”, European Commission Joint Research Centre.

Collier, P., Venables, A.J. (2012), “Greening Africa? Technologies, endowments and the latecomer effect”, Energy Economics, Elsevier, vol. 34(S1), pages S75-S84.

de Rassenfosse, G., Dernis, H., Guellec, D., Picci, L. and B. van Pottelsberghe de la Potterie (2013) "The worldwide count of priority patents: A new indicator of inventive activity", Research Policy, Volume 42, Issue 3, Pages 720-737.

Dechezleprêtre, A., M. Glachant, I. Haščič, N. Johnstone, and Y. Ménière (2011), "Invention and transfer of climate change mitigation technologies on a global scale: A study drawing on patent data," in Review of Environmental Economics and Policy, Volume 5, Issue 1, Pages 109-130.

Dernis, H. and M. Khan (2004), “Triadic patent families methodology”, STI Working Paper 2004/2. OECD Publishing.

Dernis, H., Guellec, D. and B. van Pottelsberghe (2001), "Using patent counts for cross-country comparisons of technology output”, STI Review No. 27, OECD Publishing.

Faust, K. (1990), "Early identIfication of Technological Advances on the Basis of Patent Data", Scientometrics, Vol. 19(5-6), pp. 473-480.

Faust, K. and H. Schedl (1983), "International Patent Data: Their Utilisation for the Analysis of Technological Developments”, World Patent Information, Vol. 5(3), pp. 144-157.

Guellec, D. and B. van Pottelsberghe de la Potterie (2000), “Applications, Grants and the Value of a Patent”, Economics Letters, Vol. 69, pp. 109-114.

Harhoff, D., F.M. Scherer and K. Vopel (2003), “Citations, family size, opposition and the value of Patent Rights”, Research Policy, Vol. 32, pp. 1343-63.

Haščič, I. and N. Johnstone (2011), "Innovation in Electric and Hybrid Vehicle Technologies: The Role of Prices, Standards and R\&D", in OECD, Invention and Transfer of Environmental Technologies, OECD Publishing. http://dx.doi.org/10.1787/9789264115620-5-en 
The Use of Patent Statistics for International Comparisons and Analysis of Narrow Technological Fields

Haščič, I. and M. Migotto (2015) "Measuring Environmental Innovation Using Patent Data: Policy

Relevance”, OECD Environment Working Papers, OECD Publishing (forthcoming).

Haščič, I., J. Silva and N. Johnstone (2012), “Climate Mitigation and Adaptation in Africa: Evidence from Patent Data”, OECD Environment Working Papers, No. 50, OECD Publishing, Paris. http://dx.doi.org/10.1787/5k8zng5smxjg-en

Haščič, I., Johnstone, N., Watson, F., Kaminker, C. (2010), “Climate Policy and Technological Innovation and Transfer: An Overview of Trends and Recent Empirical Results”, OECD Environment Working Papers, No. 30, OECD Publishing, Paris. http://dx.doi.org/10.1787/5km33bnggcd0-en

Johnstone, N., I. Haščič, J. Poirier, M. Hemar and C. Michel (2012) "Environmental policy stringency and technological innovation: evidence from survey data and patent counts", Applied Economics, Volume 44, Issue 17, 2012, pages 2157-2170.

Lanzi, E., Verdolini, E. and I. Haščič (2011) "Efficiency-improving fossil fuel technologies for electricity generation: Data selection and trends,” in Energy Policy, Volume 39, Issue 11, Pages 7000-7014.

Martinez, C. (2010), “Insight into Different Types of Patent Families”, OECD Science, Technology and Industry Working Papers, No. 2010/02, OECD Publishing, Paris.

http://dx.doi.org/10.1787/5kml97dr6ptl-en

Noailly J. and R. Smeets (2013) "Directing Technical Change from Fossil-Fuel to Renewable Energy Innovation: An Empirical Application Using Firm-Level Patent Data," FEEM Working Papers 2013.34, Fondazione Eni Enrico Mattei.

OECD (2012), Energy and Climate Policy: Bending the Technological Trajectory, OECD Studies on Environmental Innovation, OECD Publishing. http://dx.doi.org/10.1787/9789264174573-en

OECD (2011), Invention and Transfer of Environmental Technologies, OECD Studies on Environmental Innovation, OECD Publishing. http://dx.doi.org/10.1787/9789264115620-en

OECD (2009), OECD Patent Statistics Manual, OECD Publishing. http://dx.doi.org/10.1787/9789264056442en

Veefkind, V., J. Hurtado-Albir, S. Angelucci, K. Karachalios, and N. Thumm (2012), “A new EPO classification scheme for climate change mitigation technologies”, World Patent Information 34 (2): 106-111. 


\begin{abstract}
ANNEX
We consider four alternative (candidate) statistics based on different combinations of PRS codes and publication kind codes as extracted from the PRS dataset (summarized in Table A1). The order is chronological and most likely it correlates with reliability of the statistic - data obtained close to grant reflect patentee's intentions better than data gathered at the beginning of the patenting process; moreover, patentee's preferences may evolve over time.
\end{abstract}

Table A1. Candidate statistics and legal status search strategy

\begin{tabular}{lll}
\hline Candidate statistic & & Search strategy \\
\hline $\begin{array}{l}\text { 1. Propensity to designate states at } \\
\text { application }\end{array}$ & $A K-A$ & prs_code $=A K$ and publ_kind_code $=A \%$ \\
$\begin{array}{l}\text { 2. Propensity to designate states at } \\
\text { payment of fee }\end{array}$ & $A K X-R B V$ & prs_code $=A K X$ or prs_code $=R B V$ \\
$\begin{array}{l}\text { 3. Propensity to designate states at } \\
\text { grant }\end{array}$ & AK-B & prs_code $=A K$ and publ_kind_code $=B \%$ \\
$\begin{array}{l}\text { 4. Propensity to pay post-grant fees } \\
\text { (annual maintenance fees) }\end{array}$ & PGFP & prs_code $=P G F P$ \\
\hline
\end{tabular}

While the data underlying statistic \#3 seem as a suitable option, there seems to be a problem of scale. Three countries have propensities that approach (or exceed) 90\% (Germany, France, UK). While one would expect that these countries rank among the most often designated countries, the propensities suggested here are surprisingly high. Moreover, several rather minor economies show rather high propensities (e.g. Estonia has propensity almost as high as the Czech Republic, and Cyprus is not far from Denmark). This indicates that patentees might designate many countries at grant but then do not translate the application in the local language (validation) neither pay maintenance fees. Given this, statistic \#4 is the best option. 